\title{
Measurement of the Jet Mass Distribution and Top Quark Mass in Hadronic Decays of Boosted Top Quarks in $p p$ Collisions at $\sqrt{s}=13 \mathrm{TeV}$
}

\author{
A. M. Sirunyan et al." \\ (CMS Collaboration)
}

(Received 9 November 2019; revised manuscript received 25 March 2020; accepted 1 May 2020; published 21 May 2020)

\begin{abstract}
A measurement is reported of the jet mass distribution in hadronic decays of boosted top quarks produced in $p p$ collisions at $\sqrt{s}=13 \mathrm{TeV}$. The data were collected with the CMS detector at the LHC and correspond to an integrated luminosity of $35.9 \mathrm{fb}^{-1}$. The measurement is performed in the lepton + jets channel of $t \bar{t}$ events, where the lepton is an electron or muon. The products of the hadronic top quark decay $t \rightarrow b W \rightarrow b q \bar{q}^{\prime}$ are reconstructed as a single jet with transverse momentum larger than $400 \mathrm{GeV}$. The $t \bar{t}$ cross section as a function of the jet mass is unfolded at the particle level and used to extract a value of the top quark mass of $172.6 \pm 2.5 \mathrm{GeV}$. A novel jet reconstruction technique is used for the first time at the LHC, which improves the precision by a factor of 3 relative to an earlier measurement. This highlights the potential of measurements using boosted top quarks, where the new technique will enable future precision measurements.
\end{abstract}

DOI: 10.1103/PhysRevLett.124.202001

The top quark is the most massive known elementary particle. Its large mass $m_{t}$ leads to significant contributions from quantum corrections to the mass of the Higgs boson and precision observables in the electroweak sector. As a consequence, the top quark plays an important role in the mechanism of electroweak symmetry breaking. Precision measurements of $m_{t}$ provide a crucial input for consistency checks of the standard model [1,2]. Direct measurements of $m_{t}$ at the CERN LHC reach a precision of around $0.5 \mathrm{GeV}$ [3-9]. However, an ambiguity in the interpretation of the results originates from the modeling of partonshower dynamics and nonperturbative effects in quantum chromodynamics (QCD). The result can depend on the Monte Carlo (MC) event generator, the tuning of its free parameters, and the observables used [10]. Precisely relating the experimentally obtained value of $m_{t}$ to the pole mass or a mass in another well-defined renormalization scheme is therefore difficult from first principles [11].

As an alternative, a value of the pole mass can be extracted through measurements of the total $[12-14,14,15]$ and differential $[16,17] t \bar{t}$ production cross sections, with a precision of approximately $1 \mathrm{GeV}$. These measurements are dominated by $t \bar{t}$ threshold production, where uncertainties due to parton distribution functions (PDFs) and higherorder QCD corrections are important [18-20]. Another

\footnotetext{
*Full author list given at the end of the article.

Published by the American Physical Society under the terms of the Creative Commons Attribution 4.0 International license. Further distribution of this work must maintain attribution to the author(s) and the published article's title, journal citation, and DOI. Funded by SCOAP ${ }^{3}$.
}

way to determine $m_{t}$ involves measuring top quarks produced with large Lorentz boosts, where the decay products $t \rightarrow b W \rightarrow b q \bar{q}^{\prime}$ are contained in a single jet. The jet mass $\left(m_{\mathrm{jet}}\right)$ peak location is sensitive to $m_{t}$ and can be calculated from first principles [21-27] in soft-collinear effective theory [28-31].

A past measurement reporting the $t \bar{t}$ cross section as a function of $m_{\mathrm{jet}}$ in the $\ell+$ jets final state, where $\ell$ is an electron or muon, was carried out in proton-proton $(p p)$ collisions at $\sqrt{s}=8 \mathrm{TeV}$ [32]. This Letter reports a new measurement of the $m_{\text {jet }}$ distribution in $p p$ collisions at $13 \mathrm{TeV}$ using several important improvements, including jet clustering with the XCone algorithm [33], used for the first time in an LHC analysis, and an improved unfolding procedure using sideband regions with high granularity.

The central feature of the CMS apparatus is a superconducting solenoid of $6 \mathrm{~m}$ internal diameter, providing a magnetic field of $3.8 \mathrm{~T}$. A silicon pixel and strip tracker, a lead tungstate crystal electromagnetic calorimeter (ECAL), and a brass and scintillator hadron calorimeter, each composed of a central barrel and two end sections, reside within the solenoid volume. Forward calorimeters extend the pseudorapidity $(\eta)$ coverage provided by the barrel and end detectors. Muons are detected in gas-ionization chambers embedded in the steel flux-return yoke outside the solenoid. A more detailed description of the CMS detector, together with a definition of the coordinate system, can be found in Ref. [34]. The particle-flow (PF) algorithm [35] aims to reconstruct and identify each individual particle in an event, using an optimized combination of information from the various elements of the CMS detector. The candidate vertex with the largest sum of the square of 
the transverse momenta $p_{T}^{2}$ of the physics objects is taken to be the primary $p p$ interaction vertex; more details are given in Sec. 9.4.1 in Ref. [36]. From PF candidates, jets are reconstructed using the anti- $k_{T}$ [37] or the XCone [33] algorithm as implemented in the FASTJET software package [38]. The anti- $k_{T}$ jets are obtained using a distance parameter of 0.4. In the jet-clustering procedure, charged PF candidates are excluded if they are associated to vertices from additional inelastic $p p$ interactions within the same bunch crossing (pileup).

The POWHEG [39-44] v2 generator is used for simulating $t \bar{t}$ production at next-to-leading order (NLO). Alternatively, $t \bar{t}$ production is simulated with MadGraph5_aMC@NLO v2.2.2 $[45,46]$ at NLO to check a potential generator dependence of the measured cross sections. Background events resulting from the production of single top quarks are also generated in POWHEG at NLO, where spin correlations are taken into account [47]. The production of a $\mathrm{W}$ boson with additional jets is simulated using MadGraph5_aMC@NLO at NLO. Events from Drell-Yan (DY) production with additional jets are simulated in MadGraph5_aMC@NLO at leading order (LO) and are normalized to the next-to-next-toleading-order cross section [48]. The simulation of the production of two heavy gauge bosons with additional jets is performed at LO with PYTHIA v8.212 [49]. Events in which jets are produced only through QCD interactions are also simulated with PYTHIA at LO.

In simulated MadGraph5_aMC@NLO events, the matrix element (ME) calculations at NLO and LO accuracy are matched to parton showers with the FxFx [50] and MLM [51] algorithms, respectively. The parton shower, hadronization process, and multiple-parton interactions are simulated using PYTHIA. The NNPDF3.0 [52] PDFs at LO and NLO are used for the respective processes simulated at LO and NLO. The UE tune CUETP8M2T4 [53] is used to simulate $t \bar{t}$ and single top quark production in the $t$ channel; all other processes are simulated using CUETP8M1 [54,55]. The detector response is simulated with the GEANT4 package [56,57]. Simulated events are processed through the software chain used for collision data and are reweighted to match the observed distribution in the number of pileup interactions in the data.

This analysis uses data recorded with the CMS detector that correspond to an integrated luminosity of $35.9 \mathrm{fb}^{-1}$ [58]. Events containing the decay of a top quark to a final state including a muon are selected using a single-muon trigger [59] that requires the presence of at least one muon candidate with a transverse momentum $p_{T}>50 \mathrm{GeV}$ and $|\eta|<2.4$. For events containing a final-state electron, the trigger requires the presence of at least one isolated candidate with $p_{T}>27 \mathrm{GeV}$, or an electron candidate without an isolation requirement but with $p_{T}>115 \mathrm{GeV}$ and $|\eta|<2.5$, or at least one photon candidate with $p_{T}>$ $175 \mathrm{GeV}$ and $|\eta|<2.5$. The latter requirement ensures that events containing electrons with high $p_{T}$ are selected with high efficiency.
Lepton candidates (electrons or muons) must have $p_{T}>55 \mathrm{GeV},|\eta|<2.4$. Following the requirement at the trigger level, electrons with $p_{T}<120 \mathrm{GeV}$ must pass an isolation requirement [60], where the isolation is defined as the $p_{T}$ sum of charged hadrons and neutral particles in a cone with radius $\Delta R=0.3$ around the electron. The angular distance between two objects is defined as $\Delta R=\sqrt{(\Delta \eta)^{2}+(\Delta \phi)^{2}}$, where $\phi$ is the azimuthal angle in radians. Electrons with $p_{T}>120 \mathrm{GeV}$ and muons with $p_{T}>55 \mathrm{GeV}$ are required to pass a twodimensional selection of either $\Delta R(\ell, j)>0.4$ or $p_{T, \text { rel }}(\ell, j)>40 \mathrm{GeV}$, where $j$ is the anti- $k_{T}$ jet with minimal angular separation $\Delta R$ from the lepton $\ell$ and $p_{T, \text { rel }}(\ell, j)$ is the component of the lepton momentum orthogonal to the anti- $k_{T}$-jet axis $[61,62]$. Each selected event must contain a single lepton.

The XCone jets are obtained through a two-step jet clustering [63]. First, the exclusive XCone algorithm is applied with a distance parameter of $R_{\text {jet }}=1.2$ and the specification of returning two jets, corresponding to the two boosted top quarks in the event. Using the constituents of these two large jets as input, XCone is run again with the distance parameter $R_{\text {sub }}=0.4$ and the parameter of the number of subjets in each jet $N_{\text {sub }}=3$. Subjets are considered only if they are within $|\eta|<2.4$. This procedure results in exactly two large-radius XCone jets with three $\mathrm{XCone}$ subjets each. The final result is not influenced by the number of subjets within the large XCone jet including the lepton, where $N_{\text {sub }}=2$ would be the natural choice for clustering the visible products of the decay $t \rightarrow b W \rightarrow b \ell \nu$. The four-momentum of the lepton candidate is subtracted from the four-momentum of the anti- $k_{T}$ jet or XCone subjet if $\Delta R(\ell, j)<0.4$. Jet energy corrections [64] derived for anti- $k_{T}$ jets are applied to anti- $k_{T}$ jets and XCone subjets. The jet energy resolution in simulated events is smeared to match the resolution in data. An additional correction applied to the XCone-subjet momenta is obtained from simulated $t \bar{t}$ events in the all-jets channel to account for differences between the XCone-subjet momenta and the momenta of anti- $k_{T}$ jets. This correction is parametrized as a function of XCone subjet $p_{T}$ and $|\eta|$ and has an average size of $2 \%$, with an average uncertainty of $0.3 \%$.

The four-momenta of the three XCone subjets are combined to form the final XCone jet. The XCone jet used to perform the measurement is the one with the largest distance $\Delta R$ to the selected lepton. Each of the three XCone subjets in this jet must have $p_{T}>30 \mathrm{GeV}$. The XCone-jet mass $m_{\text {jet }}$ is the invariant mass of all PF candidates clustered into the three XCone subjets.

In order to identify jets originating from the hadronization of $b$ quarks, the combined secondary vertex v2 (CSVv2) [65] algorithm is applied to the anti- $k_{T}$ jets. These candidate $\mathrm{b}$ jets are required to have $p_{T}>30 \mathrm{GeV}$ and $|\eta|<2.4$ and must pass the tight working point of the CSVv2 algorithm. 
The fiducial region chosen for this measurement is studied using simulations at the particle level, defined by all particles with average lifetimes longer than $10^{-8} \mathrm{~s}$. The kinematic phase space of this region is defined through $t \bar{t}$ events containing one lepton with $p_{T}^{\ell}>60 \mathrm{GeV}$, which originates from the decay of a $W$ boson; the $\tau$ lepton decays are not considered part of the signal. Particle-level jets are obtained with a clustering identical to the one in the data. The particle-level XCone jet with largest distance $\Delta R$ to the lepton is required to have $p_{T}>400 \mathrm{GeV}$, and each of its XCone subjets must have $p_{T}>30 \mathrm{GeV}$. Its mass has to be greater than the mass obtained by summing the fourmomenta of the second-highest XCone jet in $p_{T}$ and the lepton. The resulting distribution in $m_{\text {jet }}$ at the particle level has a width half as large as for Cambridge-Aachen (CA) jets [66,67] with $R_{\text {jet }}=1.2$, as used in a previous measurement [32]. The improvement is due to the two-step $\mathrm{XCone}$ jet clustering procedure, which acts as a grooming algorithm [68-70], similar to trimming [71], on the large jet. The advantage of XCone over other grooming algorithms in this measurement is its dynamical interpolation between the resolved and boosted regime, i.e., between three well-separated subjets and three subjets close together, which would not be resolved by other reconstruction methods.

At the reconstruction level, the same criteria are used as in the definition of the fiducial phase space at the particle level. In addition, at the reconstruction level, an event has to have at least one b-tagged anti- $k_{T}$ jet and $p_{T}^{\text {miss }}>50 \mathrm{GeV}$, which suppresses non- $t \bar{t}$ backgrounds. Here, $p_{T}^{\text {miss }}$ is the magnitude of the negative vector sum of the transverse momenta of the PF candidates in an event [72]. The resulting $m_{\text {jet }}$ distribution for XCone jets with $p_{T}^{\text {jet }}>400 \mathrm{GeV}$ is displayed in Fig. 1. Backgrounds originate from singly produced top quarks and from $W+$ jets events. Contributions from DY + jets, diboson, and QCD multijet production are found to be negligible. The $t \bar{t}$ simulation is scaled, such that the number of simulated events matches the number of backgroundsubtracted events in the data. The distribution shows a pronounced and narrow peak close to the value of $m_{t}$. The $\mathrm{XCone-jet} \mathrm{reconstruction} \mathrm{results} \mathrm{in} \mathrm{a} \mathrm{large} \mathrm{improvement}$ of the experimental resolution in $m_{\text {jet }}$. With XCone, a resolution of $6 \%$ is achieved, compared to a resolution of approximately $14 \%$ for CA jets with $R_{\text {jet }}=1.2$.

The measurement at the particle level uses a regularized unfolding procedure based on a least-squares fit, implemented in the TUnfold [73] framework. The optimal regularization strength is determined through a minimization of the average global correlation coefficient in the output bins [74]. The response matrix is evaluated by using $t \bar{t}$ events simulated with POWHEG that pass the particle- or reconstruction-level requirements. Prior to the unfolding, contributions from background processes are subtracted

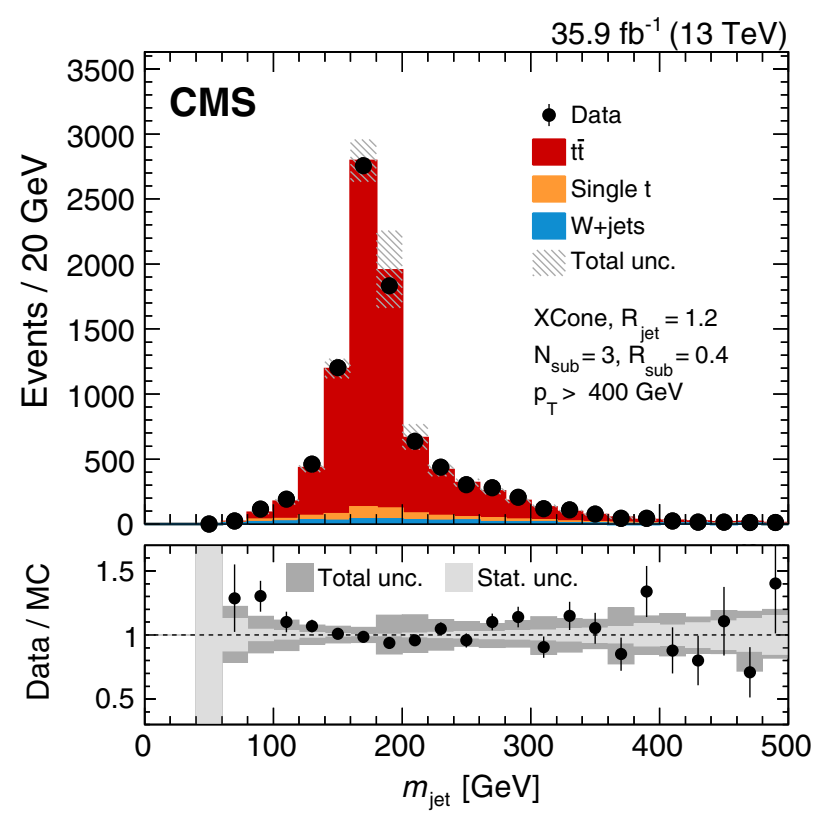

FIG. 1. Reconstructed distribution of $m_{\text {jet }}$ after the full event selection in the $\ell+$ jets channel. The vertical bars on the points show the statistical uncertainty. The hatched region shows the total uncertainty in the simulation, including the statistical and experimental systematic uncertainties. The lower panel shows the ratio of the data to the simulation. The uncertainty band includes the statistical and experimental systematic uncertainties, where the statistical (light gray) and total (dark gray) uncertainties are shown separately in the ratio.

from data. Sideband regions are included in the unfolding process to constrain migrations into and out of the measurement phase space. Five sideband regions are defined by the requirements: $55<p_{T}^{\ell}<60 \mathrm{GeV}, 350<p_{T}^{\text {jet }}<$ $400 \mathrm{GeV}$, at least one XCone subjet with $p_{T}<30 \mathrm{GeV}$, $m_{\text {jet }}$ less than the mass of the second XCone jet and lepton system, and at least one anti- $k_{T}$ jet passing a looser b-tagging requirement with no anti- $k_{T}$ jet passing the tight b-tagging requirement. In addition, the measurement region is divided into three bins in $p_{T}^{\text {jet }}$. Except for the sideband with a looser $b$ tag, all sideband selections have corresponding selections at the particle level in the evaluation of the migration matrix. In this matrix, the number of bins in $m_{\text {jet }}$ at the particle level is larger than the number of bins in which the final measurement is presented. This helps to reduce the dependence on variations in signal modeling through a more precise determination of migration effects. The electron and muon channels are combined before the unfolding to increase the statistical precision but are also unfolded separately to verify their consistency.

Experimental uncertainties are estimated using simulation and propagated through the unfolding process. We consider uncertainties in the pileup reweighting [75], trigger, lepton identification and b-tagging [65] efficiencies, and also those related to the jet energy scale [64] and 
jet energy resolution for anti- $k_{T}$ jets and XCone subjets, and additional XCone-subjet corrections. Uncertainties related to the integrated luminosity [58] and the production cross sections of all significant background processes [7681] are also included. Uncertainties arising from choices in modeling the signal include changes made in renormalization and factorization scales $\mu_{R}$ and $\mu_{F}$, changes in $m_{t}$ by $\pm 3 \mathrm{GeV}$, changes in PDFs, and choices in modeling of parton showers (PS) and their matching to the ME calculation and the underlying event (UE). Uncertainties in the modeling of PS include changes in scales of initialand final-state radiation (ISR and FSR, respectively) and changes in the ME matching parameter $h_{\text {damp }}$ [53]. The uncertainty related to modeling the UE is estimated by changing the model of color reconnection in PYTHIA [82] and using two other schemes $[83,84]$. Uncertainties from modeling $b$ quark fragmentation and the semileptonic branching fractions of $\mathrm{b}$ hadrons are found to be negligible.

The measured differential cross section in the data is shown in Fig. 2 (top) and compared to the predictions from POWHEG and MadGraph5_aMC@NLO with $m_{t}=172.5 \mathrm{GeV}$. In the peak region, the total relative uncertainty is between $16 \%$ and $36 \%$, of which the dominant contribution is $12 \%-31 \%$ from the jet energy scale uncertainty. The largest model uncertainty is from FSR modeling, with an uncertainty of $4 \%-18 \%$. The statistical uncertainty is $6 \%-7 \%$. The total measured $t \bar{t}$ cross section in the fiducial region of $112<m_{\text {jet }}<232 \mathrm{GeV}$ is $\sigma=527 \pm 15$ (stat) $\pm 39(\exp ) \pm$ 29 (model) fb. The cross section predicted by POWHEG is $680 \pm 109 \mathrm{fb}$, where the theoretical uncertainty is obtained by changing the scales $\mu_{R}$ and $\mu_{F}$, the ISR and FSR PS scales, the parameter $h_{\mathrm{damp}}$, and the UE modeling in the simulation. A smaller cross section is observed in the data relative to the simulation, in agreement with previous high$p_{T}$ top quark measurements [32,85-88].

Figure 2 (bottom) shows the normalized differential cross section as a function of $m_{\text {jet }}$, which is obtained by dividing the differential cross section by the total cross section in the fiducial region. The normalized differential cross section benefits from a partial cancellation of systematic uncertainties and shows good agreement with the prediction from POWHEG for a value of $m_{t}=172.5 \mathrm{GeV}$.

The normalized differential cross section can be used to extract a value of $m_{t}$. A fit is performed based on the $\chi^{2}$ evaluated as $\chi^{2}=d^{T} V^{-1} d$, where $d$ is the vector of differences between the measured normalized cross sections and the predictions obtained from POWHEG for different values of $m_{t}$. The symbol $V$ represents the covariance matrix that contains statistical, experimental systematic, signal modeling in the unfolding, and theoretical uncertainties. The result is

$$
\begin{aligned}
m_{t}= & 172.6 \pm 0.4(\text { stat }) \pm 1.6(\exp ) \\
& \pm 1.5(\text { model }) \pm 1.0(\text { theo }) \mathrm{GeV} .
\end{aligned}
$$
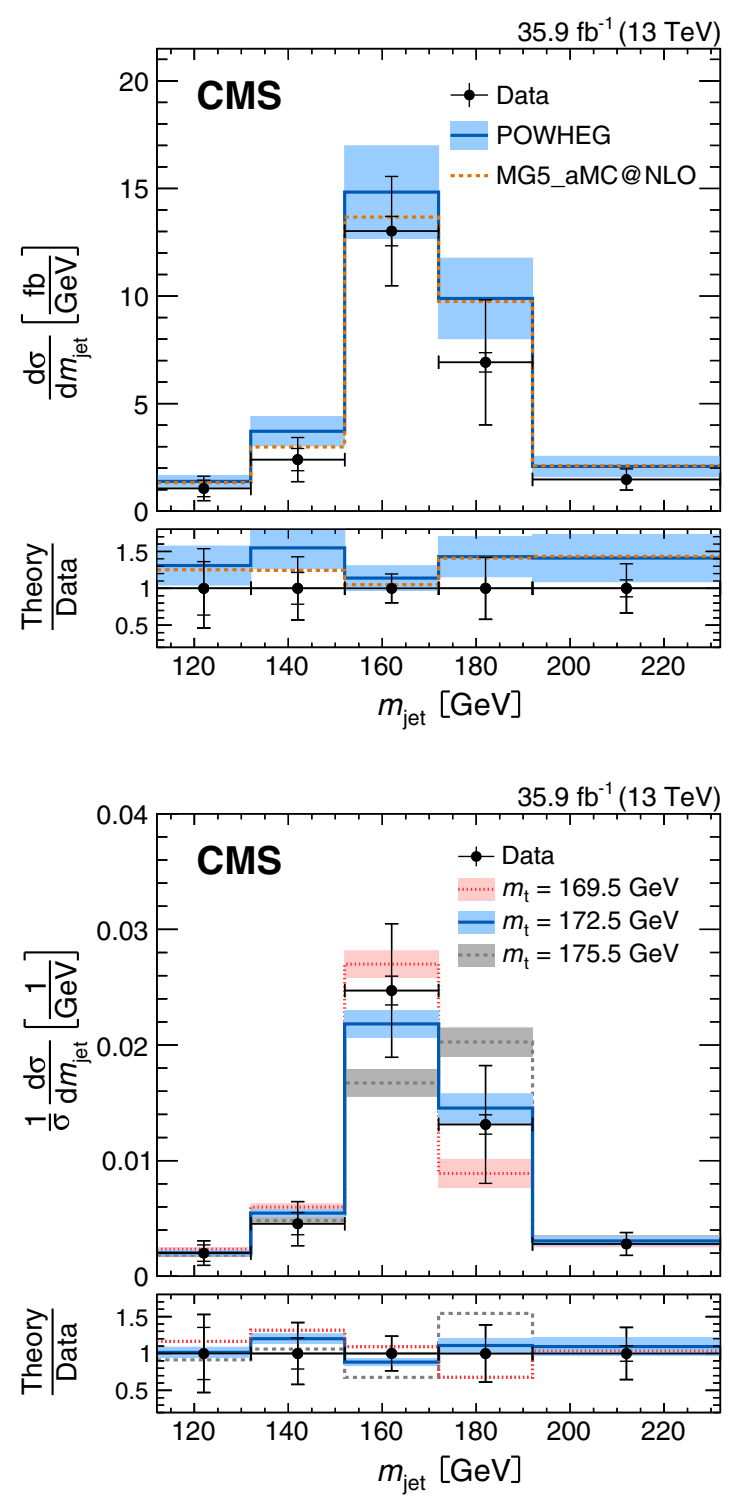

FIG. 2. The particle-level $t \bar{t}$ differential cross section in the fiducial region as a function of the XCone-jet mass (top). The measurement is compared to predictions from POWHEG and MadGraph5_aMC@NLO with $m_{t}=172.5 \mathrm{GeV}$. Theoretical uncertainties are shown as bands for the predictions from POWHEG. The normalized differential cross section (bottom) is compared to predictions from POWHEG for different values of $m_{t}$. The vertical bars represent the statistical (inner) and the total (outer) uncertainties. The horizontal bars reflect the bin widths. The lower panels show the ratios of theoretical predictions to data.

This result is a determination of $m_{t}$ from decays of boosted top quarks, with an average energy scale of approximately $480 \mathrm{GeV}$, much larger than the scale in $m_{t}$ measurements from threshold production. The improvement in precision by a factor of 3.6 relative to the measurement at $8 \mathrm{TeV}$ [32] is attributed primarily to the novel jet reconstruction using XCone. The improvement by a factor of 2 in both the $m_{\text {jet }}$ width at the particle level and experimental resolution, together with more integrated luminosity and an increased 
value of $\sqrt{s}$, provides a reduction by a factor of about 14 in the statistical uncertainty.

The systematic uncertainties are also reduced through the XCone-jet reconstruction, which enables a more precise calibration of the XCone-subjet energies and a better stability against contributions from pileup and the UE. Uncertainties from modeling are reduced through the use of additional sideband regions with higher granularity in the unfolding.

In summary, a measurement has been presented of the $t \bar{t}$ differential cross section for $t \rightarrow b W \rightarrow b q \bar{q}^{\prime}$ decays of boosted top quarks as a function of the jet mass $m_{\text {jet }}$. A determination of $m_{t}$ from the normalized $m_{\text {jet }}$ distribution provides a value of $172.6 \pm 2.5 \mathrm{GeV}$, with an uncertainty close to that of events at the $t \bar{t}$ production threshold. This measurement shows for the first time the importance of boosted top quarks for extracting standard model parameters such as $m_{t}$. The differential cross section as a function of $m_{\text {jet }}$ will enable a determination of $m_{t}$ using precise analytical calculations, feasible only in the boosted regime [26]. This is an important step in understanding the ambiguities arising between the top quark pole mass and $m_{t}$ measurements at hadron colliders. The novel reconstruction technique using the XCone jet algorithm results in the accuracy necessary for precision measurements at large top quark momenta, which will become increasingly important in future work at the LHC.

We congratulate our colleagues in the CERN accelerator departments for the excellent performance of the LHC and thank the technical and administrative staffs at CERN and at other CMS institutes for their contributions to the success of the CMS effort. In addition, we gratefully acknowledge the computing centers and personnel of the Worldwide LHC Computing Grid for delivering so effectively the computing infrastructure essential to our analyses. Finally, we acknowledge the enduring support for the construction and operation of the LHC and the CMS detector provided by the following funding agencies: BMBWF and FWF (Austria); FNRS and FWO (Belgium); CNPq, CAPES, FAPERJ, FAPERGS, and FAPESP (Brazil); MES (Bulgaria); CERN; CAS, MoST, and NSFC (China); COLCIENCIAS (Colombia); MSES and CSF (Croatia); RPF (Cyprus); SENESCYT (Ecuador); MoER, ERC IUT, PUT, and ERDF (Estonia); Academy of Finland, MEC, and HIP (Finland); CEA and CNRS/IN2P3 (France); BMBF, DFG, and HGF (Germany); GSRT (Greece); NKFIA (Hungary); DAE and DST (India); IPM (Iran); SFI (Ireland); INFN (Italy); MSIP and NRF (Republic of Korea); MES (Latvia); LAS (Lithuania); MOE and UM (Malaysia); BUAP, CINVESTAV, CONACYT, LNS, SEP, and UASLP-FAI (Mexico); MOS (Montenegro); MBIE (New Zealand); PAEC (Pakistan); MSHE and NSC (Poland); FCT (Portugal); JINR (Dubna); MON, RosAtom, RAS, RFBR, and NRC KI (Russia); MESTD
(Serbia); SEIDI, CPAN, PCTI, and FEDER (Spain); MOSTR (Sri Lanka); Swiss Funding Agencies (Switzerland); MST (Taipei); ThEPCenter, IPST, STAR, and NSTDA (Thailand); TUBITAK and TAEK (Turkey); NASU (Ukraine); STFC (United Kingdom); DOE and NSF (USA).

[1] J. Haller, A. Hoecker, R. Kogler, K. Mönig, T. Peiffer, and J. Stelzer, Update of the global electroweak fit and constraints on two-Higgs-doublet models, Eur. Phys. J. C 78, 675 (2018).

[2] M. Tanabashi et al. (Particle Data Group), Review of particle physics, Phys. Rev. D 98, 030001 (2018).

[3] ATLAS Collaboration, Measurement of the top quark mass in the $t \bar{t} \rightarrow$ dilepton channel from $\sqrt{s}=8$ TeV ATLAS data, Phys. Lett. B 761, 350 (2016).

[4] ATLAS Collaboration, Top-quark mass measurement in the all-hadronic $t \bar{t}$ decay channel at $\sqrt{s}=8 \mathrm{TeV}$ with the ATLAS detector, J. High Energy Phys. 09 (2017) 118.

[5] ATLAS Collaboration, Measurement of the top quark mass in the $t \bar{t} \rightarrow$ lepton + jets channel from $\sqrt{s}=8$ TeV ATLAS data and combination with previous results, Eur. Phys. J. C 79, 290 (2019).

[6] CMS Collaboration, Measurement of the top quark mass using proton-proton data at $\sqrt{s}=7$ and $8 \mathrm{TeV}$, Phys. Rev. D 93, 072004 (2016).

[7] CMS Collaboration, Measurement of the top quark mass in the dileptonic $t \bar{t}$ decay channel using the mass observables $M_{b \ell}, M_{\mathrm{T} 2}$, and $M_{b \ell \nu}$ in $p p$ collisions at $\sqrt{s}=8 \mathrm{TeV}$, Phys. Rev. D 96, 032002 (2017).

[8] CMS Collaboration, Measurement of the top quark mass with lepton + jets final states using $p p$ collisions at $\sqrt{s}=13 \mathrm{TeV}$, Eur. Phys. J. C 78, 891 (2018).

[9] CMS Collaboration, Measurement of the top quark mass in the all-jets final state at $\sqrt{s}=13 \mathrm{TeV}$ and combination with the lepton + jets channel, Eur. Phys. J. C 79, 313 (2019).

[10] S. Ferrario Ravasio, T. Ježo, P. Nason, and C. Oleari, A theoretical study of top-mass measurements at the LHC using NLO + PS generators of increasing accuracy, Eur. Phys. J. C 78, 458 (2018).

[11] A. H. Hoang, S. Plätzer, and D. Samitz, On the cutoff dependence of the quark mass parameter in angular ordered parton showers, J. High Energy Phys. 10 (2018) 200.

[12] ATLAS Collaboration, Measurement of the $t \bar{t}$ production cross-section using $e \mu$ events with b-tagged jets in $p p$ collisions at $\sqrt{s}=7$ and $8 \mathrm{TeV}$ with the ATLAS detector, Eur. Phys. J. C 74, 3109 (2014); Eur. Phys. J. C 76, 642(A) (2016).

[13] CMS Collaboration, Measurement of the $t \bar{t}$ production cross section in the $e \mu$ channel in proton-proton collisions at $\sqrt{s}=7$ and $8 \mathrm{TeV}$, J. High Energy Phys. 08 (2016) 029 .

[14] CMS Collaboration, Measurement of the $t \bar{t}$ production cross section, the top quark mass, and the strong coupling constant using dilepton events in $p p$ collisions at $\sqrt{s}=13$ TeV, Eur. Phys. J. C 79, 368 (2019). 
[15] ATLAS Collaboration, Measurement of the $t \bar{t}$ production cross-section and lepton differential distributions in $e \mu$ dilepton events from $p p$ collisions at $\sqrt{s}=13 \mathrm{TeV}$ with the ATLAS detector, arXiv:1910.08819 [Eur. Phys. J. C (to be published)].

[16] ATLAS Collaboration, Measurement of lepton differential distributions and the top quark mass in $t \bar{t}$ production in $p p$ collisions at $\sqrt{s}=8 \mathrm{TeV}$ with the ATLAS detector, Eur. Phys. J. C 77, 804 (2017).

[17] CMS Collaboration, Measurement of $t \bar{t}$ normalised multi-differential cross sections in $p p$ collisions at $\sqrt{s}=13 \mathrm{TeV}$, and simultaneous determination of the strong coupling strength, top quark pole mass, and parton distribution functions, arXiv:1904.05237 [Eur. Phys. J. C (to be published)].

[18] N. Kidonakis, NNNLO soft-gluon corrections for the topantitop pair production cross section, Phys. Rev. D 90, 014006 (2014).

[19] M. Guzzi, K. Lipka, and S.-O. Moch, Top-quark pair production at hadron colliders: Differential cross section and phenomenological applications with DiffTop, J. High Energy Phys. 01 (2015) 082.

[20] C. Muselli, M. Bonvini, S. Forte, S. Marzani, and G. Ridolfi, Top quark pair production beyond NNLO, J. High Energy Phys. 08 (2015) 076.

[21] S. Fleming, A. H. Hoang, S. Mantry, and I. W. Stewart, Jets from massive unstable particles: Top-mass determination, Phys. Rev. D 77, 074010 (2008).

[22] S. Fleming, A. H. Hoang, S. Mantry, and I. W. Stewart, Top jets in the peak region: Factorization analysis with next-to-leading-log resummation, Phys. Rev. D 77, 114003 (2008).

[23] A. Jain, I. Scimemi, and I. W. Stewart, Two-loop jetfunction and jet-mass for top quarks, Phys. Rev. D 77, 094008 (2008).

[24] A. H. Hoang, A. Pathak, P. Pietrulewicz, and I. W. Stewart, Hard matching for boosted tops at two loops, J. High Energy Phys. 12 (2015) 059.

[25] M. Butenschoen, B. Dehnadi, A. H. Hoang, V. Mateu, M. Preisser, and I. W. Stewart, Top Quark Mass Calibration for Monte Carlo Event Generators, Phys. Rev. Lett. 117, 232001 (2016).

[26] A. H. Hoang, S. Mantry, A. Pathak, and I. W. Stewart, Extracting a short distance top mass with light grooming, Phys. Rev. D 100, 074021 (2019).

[27] A. H. Hoang, C. Lepenik, and M. Stahlhofen, Two-loop massive quark jet functions in SCET, J. High Energy Phys. 08 (2019) 112.

[28] C. W. Bauer, S. Fleming, and M. E. Luke, Summing Sudakov logarithms in $B \rightarrow X_{s}+\gamma$ in effective field theory, Phys. Rev. D 63, 014006 (2000).

[29] C. W. Bauer, S. Fleming, D. Pirjol, and I. W. Stewart, An effective field theory for collinear and soft gluons: Heavy to light decays, Phys. Rev. D 63, 114020 (2001).

[30] C. W. Bauer and I. W. Stewart, Invariant operators in collinear effective theory, Phys. Lett. B 516, 134 (2001).

[31] C. W. Bauer, D. Pirjol, and I. W. Stewart, Soft-collinear factorization in effective field theory, Phys. Rev. D 65, 054022 (2002).
[32] CMS Collaboration, Measurement of the jet mass in highly boosted $t \bar{t}$ events from $p p$ collisions at $\sqrt{s}=8 \mathrm{TeV}$, Eur. Phys. J. C 77, 467 (2017).

[33] I. W. Stewart, F. J. Tackmann, J. Thaler, C. K. Vermilion, and T. F. Wilkason, XCone: $\mathrm{N}$-jettiness as an exclusive cone jet algorithm, J. High Energy Phys. 11 (2015) 072.

[34] CMS Collaboration, The CMS experiment at the CERN LHC, J. Instrum. 3, S08004 (2008).

[35] CMS Collaboration, Particle-flow reconstruction and global event description with the CMS detector, J. Instrum. 12, P10003 (2017).

[36] CMS Collaboration, Technical proposal for the Phase-II upgrade of the Compact Muon Solenoid, CERN, CMS Technical Proposal Report No. CERN-LHCC-2015-010, CMS-TDR-15-02, 2015, http://cds.cern.ch/record/2020886.

[37] M. Cacciari, G. P. Salam, and G. Soyez, The anti- $k_{T}$ jet clustering algorithm, J. High Energy Phys. 04 (2008) 063.

[38] M. Cacciari, G. P. Salam, and G. Soyez, FAStJet user manual, Eur. Phys. J. C 72, 1896 (2012).

[39] P. Nason, A new method for combining NLO QCD with shower Monte Carlo algorithms, J. High Energy Phys. 11 (2004) 040.

[40] S. Frixione, P. Nason, and C. Oleari, Matching NLO QCD computations with parton shower simulations: The POWHEG method, J. High Energy Phys. 11 (2007) 070.

[41] S. Alioli, P. Nason, C. Oleari, and E. Re, A general framework for implementing NLO calculations in shower Monte Carlo programs: The POWHEG BOX, J. High Energy Phys. 06 (2010) 043.

[42] S. Frixione, P. Nason, and G. Ridolfi, A positive-weight next-to-leading-order Monte Carlo for heavy flavour hadroproduction, J. High Energy Phys. 09 (2007) 126.

[43] S. Alioli, P. Nason, C. Oleari, and E. Re, NLO singletop production matched with shower in POWHEG: $s$ - and t-channel contributions, J. High Energy Phys. 09 (2009) 111; Erratum, J. High Energy Phys. 02 (2010) 011.

[44] E. Re, Single-top Wt-channel production matched with parton showers using the POWHEG method, Eur. Phys. J. C 71, 1547 (2011).

[45] J. Alwall, R. Frederix, S. Frixione, V. Hirschi, F. Maltoni, O. Mattelaer, H. S. Shao, T. Stelzer, P. Torrielli, and M. Zaro, The automated computation of tree-level and next-toleading order differential cross sections, and their matching to parton shower simulations, J. High Energy Phys. 07 (2014) 079.

[46] S. Frixione and B. R. Webber, Matching NLO QCD computations and parton shower simulations, J. High Energy Phys. 06 (2002) 029.

[47] P. Artoisenet, R. Frederix, O. Mattelaer, and R. Rietkerk, Automatic spin-entangled decays of heavy resonances in Monte Carlo simulations, J. High Energy Phys. 03 (2013) 015.

[48] Y. Li and F. Petriello, Combining QCD and electroweak corrections to dilepton production in FEWZ, Phys. Rev. D 86, 094034 (2012).

[49] T. Sjöstrand, S. Ask, J. R. Christiansen, R. Corke, N. Desai, P. Ilten, S. Mrenna, S. Prestel, C. O. Rasmussen, and P. Z. Skands, An introduction to PYTHIA8.2, Comput. Phys. Commun. 191, 159 (2015). 
[50] R. Frederix and S. Frixione, Merging meets matching in MC@NLO, J. High Energy Phys. 12 (2012) 061.

[51] J. Alwall, H. S., F. Krauss, N. Lavesson, L. Lonnblad, F. Maltoni, M. L. Mangano, M. Moretti, C. G. Papadopoulos, F. Piccinini, S. Schumann, M. Treccani, J. Winter, and M. Worek, Comparative study of various algorithms for the merging of parton showers and matrix elements in hadronic collisions, Eur. Phys. J. C 53, 473 (2008).

[52] R. D. Ball et al. (NNPDF Collaboration), Parton distributions for the LHC Run II, J. High Energy Phys. 04 (2015) 040.

[53] CMS Collaboration, Extraction and validation of a new set of CMS PYTHIA8 tunes from underlying-event measurements, Eur. Phys. J. C 80, 4 (2020).

[54] CMS Collaboration, Event generator tunes obtained from underlying event and multiparton scattering measurements, Eur. Phys. J. C 76, 155 (2016).

[55] P. Skands, S. Carrazza, and J. Rojo, Tuning PYTHIA 8.1: The Monash 2013 Tune, Eur. Phys. J. C 74, 3024 (2014).

[56] S. Agostinelli et al. (GEANT4 Collaboration), GEANT4-A simulation toolkit, Nucl. Instrum. Methods Phys. Res., Sect. A 506, 250 (2003).

[57] J. Allison et al., GEANT4 developments and applications, IEEE Trans. Nucl. Sci. 53, 270 (2006).

[58] CMS Collaboration (CMS Collaboration), CMS luminosity measurements for the 2016 data taking period, CERN, CMS Physics Analysis Summary Report No. CMS-PAS-LUM17-001, 2017, http://cds.cern.ch/record/2257069.

[59] CMS Collaboration, The CMS trigger system, J. Instrum. 12, P01020 (2017).

[60] CMS Collaboration, Performance of the CMS muon detector and muon reconstruction with proton-proton collisions at $\sqrt{s}=13 \mathrm{TeV}$, J. Instrum. 13, P06015 (2018).

[61] CMS Collaboration, Search for resonant $t \bar{t}$ production in proton-proton collisions at $\sqrt{s}=13 \mathrm{TeV}$, J. High Energy Phys. 04 (2019) 031.

[62] CMS Collaboration, Search for a heavy resonance decaying to a top quark and a vector-like top quark in the lepton + jets final state in $p p$ collisions at $\sqrt{s}=13 \mathrm{TeV}$, Eur. Phys. J. C 79, 208 (2019).

[63] J. Thaler and T. F. Wilkason, Resolving boosted jets with XCone, J. High Energy Phys. 12 (2015) 051.

[64] CMS Collaboration, Jet energy scale and resolution in the CMS experiment in $p p$ collisions at $8 \mathrm{TeV}, \mathrm{J}$. Instrum. 12, P02014 (2017).

[65] CMS Collaboration, Identification of heavy-flavour jets with the CMS detector in $p p$ collisions at $13 \mathrm{TeV}, \mathrm{J}$. Instrum. 13, P05011 (2018).

[66] Y. L. Dokshitzer, G. D. Leder, S. Moretti, and B. R. Webber, Better jet clustering algorithms, J. High Energy Phys. 08 (1997) 001.

[67] M. Wobisch and T. Wengler, Hadronization corrections to jet cross sections in deep-inelastic scattering, in Monte Carlo generators for HERA physics, Hamburg, Germany (DESY, Hamburg, 1998).

[68] A. J. Larkoski, I. Moult, and B. Nachman, Jet substructure at the Large Hadron Collider: A review of recent advances in theory and machine learning, Phys. Rep. 841, 1 (2019).
[69] S. Marzani, G. Soyez, and M. Spannowsky, Looking Inside Jets (Springer Nature, Heidelberg, 2019), Vol. 958.

[70] R. Kogler et al., Jet substructure at the Large Hadron Collider: Experimental review, Rev. Mod. Phys. 91, 045003 (2019).

[71] D. Krohn, J. Thaler, and L.-T. Wang, Jet trimming, J. High Energy Phys. 02 (2010) 084.

[72] CMS Collaboration, Performance of missing transverse momentum reconstruction in proton-proton collisions at $\sqrt{s}=13 \mathrm{TeV}$ using the CMS detector, J. Instrum. 14, P07004 (2019).

[73] S. Schmitt, TUnfold: An algorithm for correcting migration effects in high energy physics, J. Instrum. 7, T10003 (2012).

[74] S. Schmitt, Data unfolding methods in high energy physics, EPJ Web Conf. 137, 11008 (2017).

[75] CMS Collaboration, Measurement of the inelastic protonproton cross section at $\sqrt{s}=13 \mathrm{TeV}$, J. High Energy Phys. 07 (2018) 161.

[76] CMS Collaboration, Measurement of the $t \bar{t}$ production cross section using events in the $e \mu$ final state in $p p$ collisions at $\sqrt{s}=13 \mathrm{TeV}$, Eur. Phys. J. C 77, 172 (2017).

[77] CMS Collaboration, Measurement of Inclusive W and Boson Production Cross Sections in $p p$ Collisions at $\sqrt{s}=8 \mathrm{TeV}$, Phys. Rev. Lett. 112, 191802 (2014).

[78] CMS Collaboration, Cross section measurement of $t$-channel single top quark production in $p p$ collisions at $\sqrt{s}=13 \mathrm{TeV}$, Phys. Lett. B 772, 752 (2017).

[79] N. Kidonakis, NNLL threshold resummation for toppair and single-top production, Phys. Part. Nucl. 45, 714 (2014).

[80] T. Gehrmann, M. Grazzini, S. Kallweit, P. Maierhöfer, A. von Manteuffel, S. Pozzorini, D. Rathlev, and L. Tancredi, $W^{+} W^{-}$Production at Hadron Colliders in Next-to-Nextto-Leading Order QCD, Phys. Rev. Lett. 113, 212001 (2014).

[81] CMS Collaboration, Measurement of the WZ production cross section in $p p$ collisions at $\sqrt{s}=13 \mathrm{TeV}$, Phys. Lett. B 766, 268 (2017).

[82] T. Sjöstrand and M. van Zijl, A multiple interaction model for the event structure in hadron collisions, Phys. Rev. D 36, 2019 (1987).

[83] S. Argyropoulos and T. Sjöstrand, Effects of color reconnection on $t \bar{t}$ final states at the LHC, J. High Energy Phys. 11 (2014) 043.

[84] J. R. Christiansen and P. Z. Skands, String formation beyond leading colour, J. High Energy Phys. 08 (2015) 003.

[85] ATLAS Collaboration, Measurement of the differential cross-section of highly boosted top quarks as a function of their transverse momentum in $\sqrt{s}=8 \mathrm{TeV}$ protonproton collisions using the ATLAS detector, Phys. Rev. D 93, 032009 (2016).

[86] ATLAS Collaboration, Measurements of $t \bar{t}$ differential cross-sections of highly boosted top quarks decaying to all-hadronic final states in $p p$ collisions at $\sqrt{s}=13 \mathrm{TeV}$ using the ATLAS detector, Phys. Rev. D 98, 012003 (2018). 
[87] CMS Collaboration, Measurement of the integrated and differential $t \bar{t}$ production cross sections for high- $p_{T}$ top quarks in $p p$ collisions at $\sqrt{s}=8 \mathrm{TeV}$, Phys. Rev. D 94, 072002 (2016).
[88] CMS Collaboration, Measurement of differential cross sections for the production of top quark pairs and of additional jets in lepton + jets events from $p p$ collisions at $\sqrt{s}=13 \mathrm{TeV}$, Phys. Rev. D 97, 112003 (2018).

A. M. Sirunyan, ${ }^{1, a}$ A. Tumasyan, ${ }^{1}$ W. Adam, ${ }^{2}$ F. Ambrogi, ${ }^{2}$ T. Bergauer, ${ }^{2}$ J. Brandstetter, ${ }^{2}$ M. Dragicevic, ${ }^{2}$ J. Erö, ${ }^{2}$ A. Escalante Del Valle, ${ }^{2}$ M. Flechl, ${ }^{2}$ R. Frühwirth, ${ }^{2, b}$ M. Jeitler, ${ }^{2, b}$ N. Krammer, ${ }^{2}$ I. Krätschmer, ${ }^{2}$ D. Liko, ${ }^{2}$ T. Madlener, ${ }^{2}$ I. Mikulec, ${ }^{2}$ N. Rad, ${ }^{2}$ J. Schieck, ${ }^{2, b}$ R. Schöfbeck, ${ }^{2}$ M. Spanring, ${ }^{2}$ D. Spitzbart, ${ }^{2}$ W. Waltenberger, ${ }^{2}$ C.-E. Wulz, ${ }^{2, b}$ M. Zarucki, ${ }^{2}$ V. Drugakov, ${ }^{3}$ V. Mossolov, ${ }^{3}$ J. Suarez Gonzalez, ${ }^{3}$ M. R. Darwish, ${ }^{4}$ E. A. De Wolf, ${ }^{4}$ D. Di Croce, ${ }^{4}$ X. Janssen, ${ }^{4}$ A. Lelek, ${ }^{4}$ M. Pieters, ${ }^{4}$ H. Rejeb Sfar, ${ }^{4}$ H. Van Haevermaet, ${ }^{4}$ P. Van Mechelen, ${ }^{4}$ S. Van Putte, ${ }^{4}$ N. Van Remortel, ${ }^{4}$ F. Blekman, ${ }^{5}$ E. S. Bols, ${ }^{5}$ S. S. Chhibra, ${ }^{5}$ J. D'Hondt, ${ }^{5}$ J. De Clercq,${ }^{5}$ D. Lontkovskyi, ${ }^{5}$ S. Lowette, ${ }^{5}$ I. Marchesini, ${ }^{5}$ S. Moortgat, ${ }^{5}$ Q. Python, ${ }^{5}$ K. Skovpen, ${ }^{5}$ S. Tavernier, ${ }^{5}$ W. Van Doninck,${ }^{5}$ P. Van Mulders, ${ }^{5}$ D. Beghin, ${ }^{6}$ B. Bilin, ${ }^{6}$ H. Brun, ${ }^{6}$ B. Clerbaux, ${ }^{6}$ G. De Lentdecker, ${ }^{6}$ H. Delannoy, ${ }^{6}$ B. Dorney, ${ }^{6}$ L. Favart, ${ }^{6}$ A. Grebenyuk, ${ }^{6}$ A. K. Kalsi, ${ }^{6}$ A. Popov, ${ }^{6}$

N. Postiau, ${ }^{6}$ E. Starling, ${ }^{6}$ L. Thomas, ${ }^{6}$ C. Vander Velde, ${ }^{6}$ P. Vanlaer, ${ }^{6}$ D. Vannerom, ${ }^{6}$ T. Cornelis, ${ }^{7}$ D. Dobur, ${ }^{7}$ I. Khvastunov, ${ }^{7, c}$ M. Niedziela, ${ }^{7}$ C. Roskas, ${ }^{7}$ D. Trocino, ${ }^{7}$ M. Tytgat, ${ }^{7}$ W. Verbeke, ${ }^{7}$ B. Vermassen, ${ }^{7}$ M. Vit, ${ }^{7}$ O. Bondu, ${ }^{8}$ G. Bruno, ${ }^{8}$ C. Caputo, ${ }^{8}$ P. David,${ }^{8}$ C. Delaere, ${ }^{8}$ M. Delcourt, ${ }^{8}$ A. Giammanco, ${ }^{8}$ V. Lemaitre,${ }^{8}$ J. Prisciandaro, ${ }^{8}$ A. Saggio, ${ }^{8}$ M. Vidal Marono, ${ }^{8}$ P. Vischia, ${ }^{8}$ J. Zobec,${ }^{8}$ F. L. Alves, ${ }^{9}$ G. A. Alves, ${ }^{9}$ G. Correia Silva, ${ }^{9}$ C. Hensel, ${ }^{9}$ A. Moraes, ${ }^{9}$ P. Rebello Teles, ${ }^{9}$ E. Belchior Batista Das Chagas, ${ }^{10}$ W. Carvalho, ${ }^{10}$ J. Chinellato, ${ }^{10, d}$ E. Coelho, ${ }^{10}$ E. M. Da Costa, ${ }^{10}$ G. G. Da Silveira, ${ }^{10, e}$ D. De Jesus Damiao, ${ }^{10}$ C. De Oliveira Martins, ${ }^{10}$ S. Fonseca De Souza,${ }^{10}$ L. M. Huertas Guativa, ${ }^{10}$ H. Malbouisson, ${ }^{10}$ J. Martins,${ }^{10, f}$ D. Matos Figueiredo, ${ }^{10}$ M. Medina Jaime,${ }^{10, g}$ M. Melo De Almeida, ${ }^{10}$ C. Mora Herrera,${ }^{10}$ L. Mundim, ${ }^{10}$ H. Nogima, ${ }^{10}$ W. L. Prado Da Silva, ${ }^{10}$ L. J. Sanchez Rosas, ${ }^{10}$ A. Santoro, ${ }^{10}$ A. Sznajder, ${ }^{10}$ M. Thiel, ${ }^{10}$ E. J. Tonelli Manganote, ${ }^{10, d}$ F. Torres Da Silva De Araujo, ${ }^{10}$ A. Vilela Pereira,${ }^{10}$ C. A. Bernardes, ${ }^{11 a}$ L. Calligaris, ${ }^{11 a}$ T. R. Fernandez Perez Tomei, ${ }^{11 \mathrm{a}}$ E. M. Gregores, ${ }^{11 \mathrm{a}, 1 \mathrm{~b}}$ D. S. Lemos, ${ }^{11 \mathrm{a}}$ P. G. Mercadante, ${ }^{11 \mathrm{a}, 1 \mathrm{~b}}$ S. F. Novaes, ${ }^{11 \mathrm{a}}$

Sandra S. Padula, ${ }^{11 a}$ A. Aleksandrov, ${ }^{12}$ G. Antchev, ${ }^{12}$ R. Hadjiiska, ${ }^{12}$ P. Iaydjiev, ${ }^{12}$ M. Misheva, ${ }^{12}$ M. Rodozov, ${ }^{12}$ M. Shopova, ${ }^{12}$ G. Sultanov, ${ }^{12}$ M. Bonchev, ${ }^{13}$ A. Dimitrov, ${ }^{13}$ T. Ivanov, ${ }^{13}$ L. Litov,${ }^{13}$ B. Pavlov,${ }^{13}$ P. Petkov, ${ }^{13}$ W. Fang,,${ }^{14, h}$ X. Gao, ${ }^{14, h}$ L. Yuan, ${ }^{14}$ M. Ahmad, ${ }^{15}$ Z. Hu, ${ }^{15}$ Y. Wang, ${ }^{15}$ G. M. Chen, ${ }^{16}$ H. S. Chen, ${ }^{16}$ M. Chen, ${ }^{16}$ C. H. Jiang, ${ }^{16}$ D. Leggat,${ }^{16}$ H. Liao, ${ }^{16}$ Z. Liu, ${ }^{16}$ A. Spiezia, ${ }^{16}$ J. Tao, ${ }^{16}$ E. Yazgan, ${ }^{16}$ H. Zhang, ${ }^{16}$ S. Zhang, ${ }^{16, i}$ J. Zhao, ${ }^{16}$ A. Agapitos, ${ }^{17}$ Y. Ban, ${ }^{17}$ G. Chen, ${ }^{17}$ A. Levin, ${ }^{17}$ J. Li,${ }^{17}$ L. Li,${ }^{17}$ Q. Li,${ }^{17}$ Y. Mao, ${ }^{17}$ S. J. Qian, ${ }^{17}$ D. Wang, ${ }^{17}$ Q. Wang,,${ }^{17}$ M. Xiao, ${ }^{18}$ C. Avila, ${ }^{19}$ A. Cabrera,${ }^{19}$ C. Florez,${ }^{19}$ C. F. González Hernández, ${ }^{19}$ M. A. Segura Delgado, ${ }^{19}$ J. Mejia Guisao, ${ }^{20}$ J. D. Ruiz Alvarez,${ }^{20}$ C. A. Salazar González, ${ }^{20}$ N. Vanegas Arbelaez, ${ }^{20}$ D. Giljanović, ${ }^{21}$ N. Godinovic, ${ }^{21}$ D. Lelas, ${ }^{21}$ I. Puljak, ${ }^{21}$ T. Sculac, ${ }^{21}$ Z. Antunovic, ${ }^{22}$ M. Kovac, ${ }^{22}$ V. Brigljevic, ${ }^{23}$ D. Ferencek, ${ }^{23}$ K. Kadija, ${ }^{23}$ B. Mesic, ${ }^{23}$ M. Roguljic, ${ }^{23}$ A. Starodumov, ${ }^{23, j}$ T. Susa, ${ }^{23}$ M. W. Ather ${ }^{24}$ A. Attikis, ${ }^{24}$ E. Erodotou, ${ }^{24}$ A. Ioannou, ${ }^{24}$ M. Kolosova, ${ }^{24}$ S. Konstantinou, ${ }^{24}$ G. Mavromanolakis, ${ }^{24}$ J. Mousa, ${ }^{24}$ C. Nicolaou, ${ }^{24}$ F. Ptochos, ${ }^{24}$ P. A. Razis, ${ }^{24}$ H. Rykaczewski, ${ }^{24}$ D. Tsiakkouri, ${ }^{24}$ M. Finger, ${ }^{25, \mathrm{k}}$ M. Finger Jr., ${ }^{25, \mathrm{k}}$ A. Kveton, ${ }^{25}$ J. Tomsa, ${ }^{25}$ E. Ayala, ${ }^{26}$ E. Carrera Jarrin, ${ }^{27}$ A. A. Abdelalim, ${ }^{28,1, \mathrm{~m}}$ S. Abu Zeid ${ }^{28, n}$ S. Bhowmik, ${ }^{29}$ A. Carvalho Antunes De Oliveira, ${ }^{29}$ R. K. Dewanjee, ${ }^{29}$ K. Ehataht, ${ }^{29}$ M. Kadastik, ${ }^{29}$ M. Raidal, ${ }^{29}$ C. Veelken, ${ }^{29}$ P. Eerola,${ }^{30}$ L. Forthomme,${ }^{30}$ H. Kirschenmann, ${ }^{30}$ K. Osterberg, ${ }^{30}$ M. Voutilainen,${ }^{30}$ F. Garcia, ${ }^{31}$ J. Havukainen, ${ }^{31}$ J. K. Heikkilä, ${ }^{31}$ V. Karimäki, ${ }^{31}$ M. S. Kim, ${ }^{31}$ R. Kinnunen, ${ }^{31}$ T. Lampén, ${ }^{31}$ K. Lassila-Perini, ${ }^{31}$ S. Laurila, ${ }^{31}$ S. Lehti ${ }^{31}$ T. Lindén, ${ }^{31}$ P. Luukka, ${ }^{31}$ T. Mäenpää, ${ }^{31}$ H. Siikonen, ${ }^{31}$ E. Tuominen, ${ }^{31}$ J. Tuominiemi, ${ }^{31}$ T. Tuuva,${ }^{32}$ M. Besancon, ${ }^{33}$ F. Couderc, ${ }^{33}$ M. Dejardin, ${ }^{33}$ D. Denegri, ${ }^{33}$ B. Fabbro, ${ }^{33}$ J. L. Faure, ${ }^{33}$ F. Ferri, ${ }^{33}$ S. Ganjour, ${ }^{33}$

A. Givernaud, ${ }^{33}$ P. Gras, ${ }^{33}$ G. Hamel de Monchenault, ${ }^{33}$ P. Jarry, ${ }^{33}$ C. Leloup,${ }^{33}$ B. Lenzi,${ }^{33}$ E. Locci, ${ }^{33}$ J. Malcles ${ }^{33}$ J. Rander, ${ }^{33}$ A. Rosowsky, ${ }^{33}$ M. Ö. Sahin, ${ }^{33}$ A. Savoy-Navarro, ${ }^{33,0}$ M. Titov,${ }^{33}$ S. Ahuja ${ }^{34}$ C. Amendola, ${ }^{34}$ F. Beaudette, ${ }^{34}$ P. Busson, ${ }^{34}$ C. Charlot,${ }^{34}$ B. Diab,${ }^{34}$ G. Falmagne, ${ }^{34}$ R. Granier de Cassagnac, ${ }^{34}$ I. Kucher, ${ }^{34}$ A. Lobanov, ${ }^{34}$ C. Martin Perez, ${ }^{34}$ M. Nguyen, ${ }^{34}$ C. Ochando, ${ }^{34}$ P. Paganini, ${ }^{34}$ J. Rembser, ${ }^{34}$ R. Salerno, ${ }^{34}$ J. B. Sauvan, ${ }^{34}$ Y. Sirois ${ }^{34}$ A. Zabi, ${ }^{34}$ A. Zghiche, ${ }^{34}$ J.-L. Agram, ${ }^{35, p}$ J. Andrea, ${ }^{35}$ D. Bloch, ${ }^{35}$ G. Bourgatte, ${ }^{35}$ J.-M. Brom, ${ }^{35}$ E. C. Chabert, ${ }^{35}$ C. Collard, ${ }^{35}$ E. Conte, ${ }^{35, p}$ J.-C. Fontaine ${ }^{35, p}$ D. Gelé, ${ }^{35}$ U. Goerlach,${ }^{35}$ M. Jansová, ${ }^{35}$ A.-C. Le Bihan,${ }^{35}$ N. Tonon, ${ }^{35}$ P. Van Hove,${ }^{35}$ S. Gadrat,${ }^{36}$ S. Beauceron, ${ }^{37}$ C. Bernet,${ }^{37}$ G. Boudoul,${ }^{37}$ C. Camen,${ }^{37}$ A. Carle,${ }^{37}$ N. Chanon,${ }^{37}$ R. Chierici, ${ }^{37}$ D. Contardo, ${ }^{37}$ P. Depasse, ${ }^{37}$ H. El Mamouni, ${ }^{37}$ J. Fay, ${ }^{37}$ S. Gascon, ${ }^{37}$ M. Gouzevitch, ${ }^{37}$ B. Ille, ${ }^{37}$ Sa. Jain, ${ }^{37}$ F. Lagarde, ${ }^{37}$ 
I. B. Laktineh, ${ }^{37}$ H. Lattaud, ${ }^{37}$ A. Lesauvage, ${ }^{37}$ M. Lethuillier, ${ }^{37}$ L. Mirabito, ${ }^{37}$ S. Perries, ${ }^{37}$ V. Sordini,${ }^{37}$ L. Torterotot, ${ }^{37}$ G. Touquet, ${ }^{37}$ M. Vander Donckt, ${ }^{37}$ S. Viret, ${ }^{37}$ T. Toriashvili, ${ }^{38, q}$ D. Lomidze,${ }^{39}$ C. Autermann, ${ }^{40}$ L. Feld, ${ }^{40}$ M. K. Kiesel, ${ }^{40}$ K. Klein, ${ }^{40}$ M. Lipinski, ${ }^{40}$ D. Meuser, ${ }^{40}$ A. Pauls, ${ }^{40}$ M. Preuten, ${ }^{40}$ M. P. Rauch,${ }^{40}$ J. Schulz, ${ }^{40}$ M. Teroerde, ${ }^{40}$ B. Wittmer, ${ }^{40}$ M. Erdmann, ${ }^{41}$ B. Fischer, ${ }^{41}$ S. Ghosh,${ }^{41}$ T. Hebbeker ${ }^{41}$ K. Hoepfner, ${ }^{41}$ H. Keller, ${ }^{41}$ L. Mastrolorenzo, ${ }^{41}$ M. Merschmeyer,${ }^{41}$ A. Meyer, ${ }^{41}$ P. Millet, ${ }^{41}$ G. Mocellin, ${ }^{41}$ S. Mondal,${ }^{41}$ S. Mukherjee, ${ }^{41}$ D. Noll, ${ }^{41}$ A. Novak, ${ }^{41}$ T. Pook, ${ }^{41}$ A. Pozdnyakov, ${ }^{41}$ T. Quast, ${ }^{41}$ M. Radziej, ${ }^{41}$ Y. Rath, ${ }^{41}$ H. Reithler, ${ }^{41}$ J. Roemer, ${ }^{41}$ A. Schmidt, ${ }^{41}$ S. C. Schuler, ${ }^{41}$ A. Sharma, ${ }^{41}$ S. Wiedenbeck,${ }^{41}$ S. Zaleski, ${ }^{41}$ G. Flügge, ${ }^{42}$ W. Haj Ahmad, ${ }^{42, r}$ O. Hlushchenko, ${ }^{42}$ T. Kress, ${ }^{42}$ T. Müller, ${ }^{42}$ A. Nowack, ${ }^{42}$ C. Pistone, ${ }^{42}$ O. Pooth, ${ }^{42}$ D. Roy, ${ }^{42}$ H. Sert ${ }^{42}$ A. Stahl,${ }^{42, s}$ M. Aldaya Martin, ${ }^{43}$ P. Asmuss, ${ }^{43}$ I. Babounikau, ${ }^{43}$ H. Bakhshiansohi, ${ }^{43}$ K. Beernaert, ${ }^{43}$ O. Behnke, ${ }^{43}$ A. Bermúdez Martínez, ${ }^{43}$ D. Bertsche, ${ }^{43}$ A. A. Bin Anuar, ${ }^{43}$ K. Borras, ${ }^{43, t}$ V. Botta, ${ }^{43}$ A. Campbell, ${ }^{43}$ A. Cardini, ${ }^{43}$ P. Connor, ${ }^{43}$ S. Consuegra Rodríguez, ${ }^{43}$ C. Contreras-Campana, ${ }^{43}$ V. Danilov, ${ }^{43}$ A. De Wit ${ }^{43}$ M. M. Defranchis ${ }^{43}$ C. Diez Pardos ${ }^{43}$ D. Domínguez Damiani, ${ }^{43}$ G. Eckerlin, ${ }^{43}$ D. Eckstein, ${ }^{43}$ T. Eichhorn, ${ }^{43}$ A. Elwood ${ }^{43}$ E. Eren, ${ }^{43}$ E. Gallo, ${ }^{43, u}$ A. Geiser, ${ }^{43}$ A. Grohsjean, ${ }^{43}$ M. Guthoff, ${ }^{43}$ M. Haranko, ${ }^{43}$ A. Harb, ${ }^{43}$ A. Jafari ${ }^{43}$ N. Z. Jomhari, ${ }^{43}$ H. Jung, ${ }^{43}$ A. Kasem, ${ }^{43, t}$ M. Kasemann,${ }^{43}$ H. Kaveh, ${ }^{43}$ J. Keaveney, ${ }^{43}$ C. Kleinwort, ${ }^{43}$ J. Knolle,${ }^{43}$ D. Krücker, ${ }^{43}$ W. Lange, ${ }^{43}$ T. Lenz, ${ }^{43}$ J. Lidrych, ${ }^{43}$ K. Lipka, ${ }^{43}$ W. Lohmann, ${ }^{43, v}$ R. Mankel, ${ }^{43}$ I.-A. Melzer-Pellmann, ${ }^{43}$ A. B. Meyer ${ }^{43}$ M. Meyer,${ }^{43}$ M. Missiroli, ${ }^{43}$ G. Mittag, ${ }^{43}$ J. Mnich, ${ }^{43}$ A. Mussgiller, ${ }^{43}$ V. Myronenko, ${ }^{43}$ D. Pérez Adán, ${ }^{43}$ S. K. Pflitsch, ${ }^{43}$ D. Pitzl, ${ }^{43}$ A. Raspereza, ${ }^{43}$ A. Saibel,${ }^{43}$ M. Savitskyi,${ }^{43}$ V. Scheurer, ${ }^{43}$ P. Schütze, ${ }^{43}$ C. Schwanenberger, ${ }^{43}$ R. Shevchenko, ${ }^{43}$ A. Singh, ${ }^{43}$ H. Tholen, ${ }^{43}$ O. Turkot, ${ }^{43}$ A. Vagnerini, ${ }^{43}$ M. Van De Klundert, ${ }^{43}$ R. Walsh,${ }^{43}$ Y. Wen, ${ }^{43}$ K. Wichmann, ${ }^{43}$ C. Wissing ${ }^{43}$ O. Zenaiev, ${ }^{43}$ R. Zlebcik, ${ }^{43}$ R. Aggleton, ${ }^{44}$ S. Bein, ${ }^{44}$ L. Benato, ${ }^{44}$ A. Benecke, ${ }^{44}$ V. Blobel, ${ }^{44}$ T. Dreyer, ${ }^{44}$ A. Ebrahimi, ${ }^{44}$ F. Feindt ${ }^{44}$ A. Fröhlich, ${ }^{44}$ C. Garbers ${ }^{44}$ E. Garutti, ${ }^{44}$ D. Gonzalez, ${ }^{44}$ P. Gunnellini, ${ }^{44}$ J. Haller, ${ }^{44}$ A. Hinzmann, ${ }^{44}$ A. Karavdina, ${ }^{44}$ G. Kasieczka, ${ }^{44}$ R. Klanner, ${ }^{44}$ R. Kogler, ${ }^{44}$ N. Kovalchuk, ${ }^{44}$ S. Kurz, ${ }^{44}$ V. Kutzner, ${ }^{44}$ J. Lange,${ }^{44}$ T. Lange, ${ }^{44}$ A. Malara, ${ }^{44}$ J. Multhaup, ${ }^{44}$ C. E. N. Niemeyer, ${ }^{44}$ A. Perieanu, ${ }^{44}$ A. Reimers, ${ }^{44}$ O. Rieger, ${ }^{44}$ C. Scharf, ${ }^{44}$ P. Schleper, ${ }^{44}$ S. Schumann, ${ }^{44}$ J. Schwandt, ${ }^{44}$ D. Schwarz, ${ }^{44}$ J. Sonneveld, ${ }^{44}$ H. Stadie, ${ }^{44}$ G. Steinbrück, ${ }^{44}$ F. M. Stober, ${ }^{44}$ B. Vormwald,${ }^{44}$ I. Zoi, ${ }^{44}$ M. Akbiyik, ${ }^{45}$ C. Barth, ${ }^{45}$ M. Baselga ${ }^{45}$ S. Baur, ${ }^{45}$ T. Berger, ${ }^{45}$ E. Butz,${ }^{45}$ R. Caspart, ${ }^{45}$ T. Chwalek,${ }^{45}$ W. De Boer, ${ }^{45}$

A. Dierlamm, ${ }^{45}$ K. El Morabit, ${ }^{45}$ N. Faltermann, ${ }^{45}$ M. Giffels, ${ }^{45}$ P. Goldenzweig, ${ }^{45}$ A. Gottmann, ${ }^{45}$ M. A. Harrendorf, ${ }^{45}$ F. Hartmann, ${ }^{45, \mathrm{~s}}$ U. Husemann, ${ }^{45}$ S. Kudella, ${ }^{45}$ S. Mitra, ${ }^{45}$ M. U. Mozer, ${ }^{45}$ D. Müller, ${ }^{45}$ Th. Müller, ${ }^{45}$ M. Musich, ${ }^{45}$ A. Nürnberg, ${ }^{45}$ G. Quast, ${ }^{45}$ K. Rabbertz ${ }^{45}$ M. Schröder, ${ }^{45}$ I. Shvetsov, ${ }^{45}$ H. J. Simonis, ${ }^{45}$ R. Ulrich, ${ }^{45}$ M. Wassmer, ${ }^{45}$ M. Weber, ${ }^{45}$ C. Wöhrmann, ${ }^{45}$ R. Wolf, ${ }^{45}$ G. Anagnostou, ${ }^{46}$ P. Asenov, ${ }^{46}$ G. Daskalakis, ${ }^{46}$ T. Geralis,${ }^{46}$ A. Kyriakis, ${ }^{46}$ D. Loukas, ${ }^{46}$ G. Paspalaki, ${ }^{46}$ M. Diamantopoulou, ${ }^{47}$ G. Karathanasis, ${ }^{47}$ P. Kontaxakis, ${ }^{47}$ A. Manousakis-katsikakis,${ }^{47}$ A. Panagiotou, ${ }^{47}$ I. Papavergou, ${ }^{47}$ N. Saoulidou, ${ }^{47}$ A. Stakia, ${ }^{47}$ K. Theofilatos,${ }^{47}$ K. Vellidis, ${ }^{47}$ E. Vourliotis, ${ }^{47}$ G. Bakas,${ }^{48}$ K. Kousouris, ${ }^{48}$ I. Papakrivopoulos, ${ }^{48}$ G. Tsipolitis ${ }^{48}$ I. Evangelou, ${ }^{49}$ C. Foudas, ${ }^{49}$ P. Gianneios, ${ }^{49}$ P. Katsoulis, ${ }^{49}$ P. Kokkas, ${ }^{49}$ S. Mallios, ${ }^{49}$ K. Manitara, ${ }^{49}$ N. Manthos,${ }^{49}$ I. Papadopoulos, ${ }^{49}$ J. Strologas, ${ }^{49}$ F. A. Triantis, ${ }^{49}$ D. Tsitsonis, ${ }^{49}$ M. Bartók, ${ }^{50, w}$ R. Chudasama, ${ }^{50}$ M. Csanad, ${ }^{50}$ P. Major,${ }^{50}$ K. Mandal, ${ }^{50}$ A. Mehta, ${ }^{50}$ M. I. Nagy, ${ }^{50}$ G. Pasztor, ${ }^{50}$ O. Surányi ${ }^{50}$ G. I. Veres, ${ }^{50}$ G. Bencze, ${ }^{51}$ C. Hajdu, ${ }^{51}$ D. Horvath, ${ }^{51, x}$ F. Sikler, ${ }^{51}$ T. Á. Vámi ${ }^{51}$ V. Veszpremi, ${ }^{51}$ G. Vesztergombi, ${ }^{51, \mathrm{a}, \mathrm{y}}$ N. Beni, ${ }^{52}$ S. Czellar, ${ }^{52}$ J. Karancsi, ${ }^{52, w}$ A. Makovec, ${ }^{52}$ J. Molnar, ${ }^{52}$ Z. Szillasi,${ }^{52}$ P. Raics, ${ }^{53}$ D. Teyssier,${ }^{53}$ Z. L. Trocsanyi, ${ }^{53}$ B. Ujvari, ${ }^{53}$ T. Csorgo, ${ }^{54}$ W. J. Metzger, ${ }^{54}$ F. Nemes, ${ }^{54}$ T. Novak, ${ }^{54}$ S. Choudhury, ${ }^{55}$ J. R. Komaragiri, ${ }^{55}$ P. C. Tiwari, ${ }^{55}$ S. Bahinipati, ${ }^{56, z}$ C. Kar, ${ }^{56}$ G. Kole,${ }^{56}$ P. Mal, ${ }^{56}$ V. K. Muraleedharan Nair Bindhu ${ }^{56}$ A. Nayak, ${ }^{56, a a}$ D. K. Sahoo, ${ }^{56, z}$ S. K. Swain ${ }^{56}$ S. Bansal, ${ }^{57}$ S. B. Beri, ${ }^{57}$ V. Bhatnagar, ${ }^{57}$ S. Chauhan, ${ }^{57}$ R. Chawla, ${ }^{57}$ N. Dhingra, ${ }^{57}$ R. Gupta, ${ }^{57}$ A. Kaur,${ }^{57}$ M. Kaur, ${ }^{57}$ S. Kaur, ${ }^{57}$ P. Kumari, ${ }^{57}$ M. Lohan, ${ }^{57}$ M. Meena, ${ }^{57}$ K. Sandeep,${ }^{57}$ S. Sharma, ${ }^{57}$ J. B. Singh, ${ }^{57}$ A. K. Virdi,${ }^{57}$ G. Walia, ${ }^{57}$ A. Bhardwaj, ${ }^{58}$ B. C. Choudhary, ${ }^{58}$ R. B. Garg, ${ }^{58}$ M. Gola, ${ }^{58}$ S. Keshri, ${ }^{58}$ Ashok Kumar, ${ }^{58}$ M. Naimuddin, ${ }^{58}$ P. Priyanka ${ }^{58}$ K. Ranjan, ${ }^{58}$ Aashaq Shah, ${ }^{58}$ R. Sharma, ${ }^{58}$ R. Bhardwaj, ${ }^{59, b b}$ M. Bharti, ${ }^{59, b b}$ R. Bhattacharya ${ }^{59}$ S. Bhattacharya, ${ }^{59}$ U. Bhawandeep, ${ }^{59, b b}$ D. Bhowmik, ${ }^{59}$ S. Dutta, ${ }^{59}$ S. Ghosh, ${ }^{59}$ M. Maity, ${ }^{59, c c}$ K. Mondal, ${ }^{59}$ S. Nandan, ${ }^{59}$ A. Purohit, ${ }^{59}$ P. K. Rout,${ }^{59}$ G. Saha, ${ }^{59}$ S. Sarkar, ${ }^{59}$ T. Sarkar, ${ }^{59, c c}$ M. Sharan, ${ }^{59}$ B. Singh, ${ }^{59, b b}$ S. Thakur, ${ }^{59, b b}$ P. K. Behera, ${ }^{60}$ P. Kalbhor, ${ }^{60}$ A. Muhammad, ${ }^{60}$ P. R. Pujahari, ${ }^{60}$ A. Sharma,${ }^{60}$ A. K. Sikdar, ${ }^{60}$ D. Dutta, ${ }^{61}$ V. Jha, ${ }^{61}$ V. Kumar,${ }^{61}$

D. K. Mishra, ${ }^{61}$ P. K. Netrakanti, ${ }^{61}$ L. M. Pant, ${ }^{61}$ P. Shukla, ${ }^{61}$ T. Aziz,${ }^{62}$ M. A. Bhat, ${ }^{62}$ S. Dugad, ${ }^{62}$ G. B. Mohanty, ${ }^{62}$ N. Sur, ${ }^{62}$ Ravindra Kumar Verma, ${ }^{62}$ S. Banerjee, ${ }^{63}$ S. Bhattacharya, ${ }^{63}$ S. Chatterjee ${ }^{63}$ P. Das, ${ }^{63}$ M. Guchait, ${ }^{63}$ S. Karmakar, ${ }^{63}$ S. Kumar, ${ }^{63}$ G. Majumder ${ }^{63}$ K. Mazumdar, ${ }^{63}$ N. Sahoo, ${ }^{63}$ S. Sawant, ${ }^{63}$ S. Dube,${ }^{64}$ V. Hegde, ${ }^{64}$ B. Kansal, ${ }^{64}$ A. Kapoor,${ }^{64}$

K. Kothekar, ${ }^{64}$ S. Pandey, ${ }^{64}$ A. Rane,${ }^{64}$ A. Rastogi,${ }^{64}$ S. Sharma,${ }^{64}$ S. Chenarani, ${ }^{65, d d}$ E. Eskandari Tadavani, ${ }^{65}$

S. M. Etesami, ${ }^{65 \text {,d }}$ M. Khakzad, ${ }^{65}$ M. Mohammadi Najafabadi, ${ }^{65}$ M. Naseri, ${ }^{65}$ F. Rezaei Hosseinabadi, ${ }^{65}$ M. Felcini ${ }^{66}$ M. Grunewald, ${ }^{66}$ M. Abbrescia, ${ }^{67 a 67 b}$ R. Aly, ${ }^{67 a, 67 b, e e}$ C. Calabria, ${ }^{67 a, 67 b}$ A. Colaleo, ${ }^{67 a}$ D. Creanza, ${ }^{67 a, 67 c}$ L. Cristella, ${ }^{67 a, 67 b}$ 
N. De Filippis, ${ }^{67 a, 67 c}$ M. De Palma, ${ }^{67 a, 67 b}$ A. Di Florio, ${ }^{67 a, 67 b}$ W. Elmetenawee,${ }^{67 a, 67 b}$ L. Fiore, ${ }^{67 a}$ A. Gelmi, ${ }^{67 a, 67 b}$ G. Iaselli, ${ }^{67 a, 67 \mathrm{c}}$ M. Ince, ${ }^{67 a, 67 b}$ S. Lezki, ${ }^{67 a, 67 b}$ G. Maggi, ${ }^{67 a, 67 c}$ M. Maggi, ${ }^{67 a}$ G. Miniello, ${ }^{67 a, 67 b}$ S. My, ${ }^{67 a, 67 b}$ S. Nuzzo, ${ }^{67 a, 67 b}$ A. Pompili, ${ }^{67 a, 67 b}$ G. Pugliese, ${ }^{67 a, 67 c}$ R. Radogna,${ }^{67 a}$ A. Ranieri, ${ }^{67 a}$ G. Selvaggi,${ }^{67 a, 67 b}$ L. Silvestris,${ }^{67 a}$ F. M. Simone, ${ }^{67 a, 67 b}$ R. Venditti, ${ }^{67 \mathrm{a}}$ P. Verwilligen, ${ }^{67 \mathrm{a}}$ G. Abbiendi, ${ }^{68 \mathrm{a}} \mathrm{C}$. Battilana, ${ }^{6 \mathrm{a}, 68 \mathrm{~b}}$ D. Bonacorsi, ${ }^{68 \mathrm{a}, 68 \mathrm{~b}}$ L. Borgonovi, ${ }^{68,68 \mathrm{~b}}$ S. Braibant-Giacomelli, ${ }^{68 a, 68 b}$ R. Campanini, ${ }^{68 a, 68 b}$ P. Capiluppi, ${ }^{68 a, 68 b}$ A. Castro, ${ }^{68 a, 68 b}$ F. R. Cavallo, ${ }^{68 a}$ C. Ciocca, ${ }^{68 a}$ G. Codispoti, ${ }^{68 a, 68 b}$ M. Cuffiani, ${ }^{68 a, 68 b}$ G. M. Dallavalle, ${ }^{68 a}$ F. Fabbri, ${ }^{68 a}$ A. Fanfani, ${ }^{68 a, 68 b}$ E. Fontanesi, ${ }^{68 a, 68 b}$

P. Giacomelli, ${ }^{68 \mathrm{a}}$ C. Grandi, ${ }^{68 \mathrm{a}}$ L. Guiducci, ${ }^{68 \mathrm{a}, 68 \mathrm{~b}}$ F. Iemmi, ${ }^{68 \mathrm{a}, 68 \mathrm{~b}}$ S. Lo Meo, ${ }^{68 \mathrm{a}, \mathrm{ff}}$ S. Marcellini, ${ }^{68 \mathrm{a}}$ G. Masetti, ${ }^{68 \mathrm{a}}$ F. L. Navarria, ${ }^{68 a, 68 b}$ A. Perrotta, ${ }^{68 \mathrm{a}}$ F. Primavera, ${ }^{68 \mathrm{a}, 68 \mathrm{~b}}$ A. M. Rossi, ${ }^{68 \mathrm{a}, 68 \mathrm{~b}}$ T. Rovelli, ${ }^{6 \mathrm{a}, 68 \mathrm{~b}}$ G. P. Siroli, ${ }^{68 a, 68 \mathrm{~b}}$ N. Tosi, ${ }^{68 \mathrm{a}}$ S. Albergo, ${ }^{69 a, 69 b, g g}$ S. Costa ${ }^{69 a, 69 b}$ A. Di Mattia, ${ }^{69 a}$ R. Potenza, ${ }^{69 a, 69 b}$ A. Tricomi ${ }^{69 a, 69 b, g g}$ C. Tuve,${ }^{6 a, 69 b}$ G. Barbagli, ${ }^{70 a}$ A. Cassese, ${ }^{70 a}$ R. Ceccarelli, ${ }^{70 a}$ V. Ciulli, ${ }^{70 a, 70 b}$ C. Civinini, ${ }^{70 a}$ R. D’Alessandro, ${ }^{70 a, 70 b}$ E. Focardi, ${ }^{70 a, 70 b}$ G. Latino, ${ }^{70 a, 70 b}$ P. Lenzi, ${ }^{70 a}, 70 \mathrm{~b}$ M. Meschini, ${ }^{70 a}$ S. Paoletti, ${ }^{70 a}$ G. Sguazzoni, ${ }^{70 a}$ L. Viliani, ${ }^{70 a}$ L. Benussi, ${ }^{71}$ S. Bianco, ${ }^{71}$ D. Piccolo, ${ }^{71}$ M. Bozzo ${ }^{72 a, 72 b}$ F. Ferro, ${ }^{72 a}$ R. Mulargia, ${ }^{72 a, 72 b}$ E. Robutti, ${ }^{72 a}$ S. Tosi, ${ }^{72 a, 72 b}$ A. Benaglia, ${ }^{73 a}$ A. Beschi, ${ }^{73 a, 73 b}$ F. Brivio, ${ }^{73 a, 73 b}$ V. Ciriolo, ${ }^{73 a, 73 b, s}$ S. Di Guida, ${ }^{73 a, 73 b, s}$ M. E. Dinardo, ${ }^{73 a, 73 b}$ P. Dini, ${ }^{73 a}$ S. Gennai, ${ }^{73 a}$ A. Ghezzi, ${ }^{73 a, 73 b}$ P. Govoni, ${ }^{73 a, 73 b}$

L. Guzzi, ${ }^{73 a, 73 b}$ M. Malberti, ${ }^{73 a}$ S. Malvezzi, ${ }^{73 a}$ D. Menasce, ${ }^{73 a}$ F. Monti, ${ }^{73 a, 73 b}$ L. Moroni, ${ }^{73 a}$ M. Paganoni, ${ }^{73 a, 73 b}$

D. Pedrini, ${ }^{73 a}$ S. Ragazzi, ${ }^{73 a, 73 b}$ T. Tabarelli de Fatis, ${ }^{73 a, 73 b}$ D. Zuolo, ${ }^{73 a, 73 b}$ S. Buontempo, ${ }^{74 a}$ N. Cavallo, ${ }^{74 a, 74 c}$ A. De Iorio, ${ }^{74 a, 74 b}$ A. Di Crescenzo, ${ }^{74 a, 74 b}$ F. Fabozzi, ${ }^{74 a, 74 c}$ F. Fienga, ${ }^{74 a}$ G. Galati, ${ }^{74 a}$ A. O. M. Iorio, ${ }^{74 a, 74 b}$ L. Lista, ${ }^{74 a, 74 b}$ S. Meola, ${ }^{74 a, 74 d, s}$ P. Paolucci, ${ }^{74 a, s}$ B. Rossi,${ }^{74 a}$ C. Sciacca, ${ }^{74 a, 74 b}$ E. Voevodina,${ }^{74 a, 74 b}$ P. Azzi, ${ }^{75 a}$ N. Bacchetta, ${ }^{75 a}$ D. Bisello, ${ }^{75 a, 75 b}$ A. Boletti, ${ }^{75 a, 75 b}$ A. Bragagnolo,${ }^{75 a, 75 b}$ R. Carlin, ${ }^{75 a, 75 b}$ P. Checchia, ${ }^{75 a}$ P. De Castro Manzano, ${ }^{75 a}$ T. Dorigo, ${ }^{75 a}$ U. Dosselli, ${ }^{75 a}$ F. Gasparini, ${ }^{75 a, 75 b}$ U. Gasparini, ${ }^{75 a, 75 b}$ A. Gozzelino, ${ }^{75 a}$ S. Y. Hoh,${ }^{75 a, 75 b}$ P. Lujan, ${ }^{75 a}$ M. Margoni, ${ }^{75 a, 75 b}$ A. T. Meneguzzo, ${ }^{75 a, 75 b}$ J. Pazzini, ${ }^{75 a, 75 b}$ M. Presilla, ${ }^{75 a, 75 b}$ P. Ronchese, ${ }^{75 a, 75 b}$ R. Rossin,${ }^{75 a, 75 b}$ F. Simonetto, ${ }^{75 a, 75 b}$ A. Tiko, ${ }^{75 a}$ M. Tosi, ${ }^{75 a, 75 b}$ M. Zanetti, ${ }^{75 a, 75 b}$ P. Zotto, ${ }^{75 a, 75 b}$ G. Zumerle, ${ }^{75 a, 75 b}$ A. Braghieri, ${ }^{76 a}$ D. Fiorina, ${ }^{76 a, 76 b}$ P. Montagna, ${ }^{76 a, 76 b}$ S. P. Ratti, ${ }^{76 a, 76 b}$ V. Re,${ }^{76 a}$ M. Ressegotti ${ }^{76 a, 76 b}$ C. Riccardi, ${ }^{76 a, 76 b}$ P. Salvini, ${ }^{76 a}$ I. Vai, ${ }^{76 a}$ P. Vitulo, ${ }^{76 a, 76 b}$ M. Biasini, ${ }^{77,77 b}$ G. M. Bilei, ${ }^{77 a}$ D. Ciangottini, ${ }^{77 a, 77 b}$ L. Fanò, ${ }^{77 a, 77 b}$ P. Lariccia, ${ }^{77 a, 77 b}$ R. Leonardi, ${ }^{77 a, 77 b}$ E. Manoni, ${ }^{77 a}$ G. Mantovani, ${ }^{77,77 b}$ V. Mariani, ${ }^{77 a, 77 b}$ M. Menichelli, ${ }^{77 a}$ A. Rossi, ${ }^{77 a, 77 b}$ A. Santocchia, ${ }^{77 a, 77 b}$ D. Spiga, ${ }^{77 a}$ K. Androsov, ${ }^{78 a}$ P. Azzurri, ${ }^{78 a}$ G. Bagliesi, ${ }^{78 a}$ V. Bertacchi, ${ }^{78 a, 78 c}$ L. Bianchini, ${ }^{78 a}$ T. Boccali, ${ }^{78 a}$ R. Castaldi, ${ }^{78 a}$ M. A. Ciocci ${ }^{78 a, 78 b}$ R. Dell'Orso, ${ }^{78 a}$ S. Donato, ${ }^{78 a}$ G. Fedi, ${ }^{78 a}$ L. Giannini, ${ }^{78 a}{ }^{78 c}$ A. Giassi, ${ }^{78 a}$ M. T. Grippo, ${ }^{78 a}$ F. Ligabue ${ }^{78 a, 78 c}$ E. Manca, ${ }^{78 a, 78 c}$ G. Mandorli, ${ }^{78 a}, 78 c$ A. Messineo, ${ }^{78 a, 78 b}$ F. Palla, ${ }^{78 a}$ A. Rizzii, ${ }^{78 a, 78 b}$ G. Rolandi, ${ }^{78 a, h h}$ S. Roy Chowdhury, ${ }^{78 a}$ A. Scribano, ${ }^{78 a}$ P. Spagnolo, ${ }^{78 a}$ R. Tenchini, ${ }^{78 a}$ G. Tonelli, ${ }^{78 a}, 78 b$ N. Turini, ${ }^{78 a}$ A. Venturi, ${ }^{78 a}$ P. G. Verdini, ${ }^{78 a}$ F. Cavallari, ${ }^{79 a}$ M. Cipriani, ${ }^{79 a, 79 b}$ D. Del Re, ${ }^{79 a, 79 b}$ E. Di Marco, ${ }^{79 a, 79 b}$ M. Diemoz, ${ }^{79 a}$ E. Longo, ${ }^{79 a, 79 b}$ P. Meridiani, ${ }^{79 a}$ G. Organtini, ${ }^{79 a, 79 b}$ F. Pandolfi, ${ }^{79 a}$ R. Paramatti, ${ }^{7 a, 79 b}$ C. Quaranta, ${ }^{79 a, 79 b}$ S. Rahatlou, ${ }^{79 a, 79 b}$ C. Rovelli, ${ }^{79 a}$ F. Santanastasio, ${ }^{79 a, 79 b}$ L. Soffi, ${ }^{79 a, 79 b}$ N. Amapane,${ }^{80 a, 80 b}$ R. Arcidiacono, ${ }^{80 a, 80 c}$ S. Argiro, ${ }^{80 a, 80 b}$ M. Arneodo, ${ }^{80 a, 80 c}$ N. Bartosik, ${ }^{80 \mathrm{a}}$ R. Bellan, ${ }^{80 \mathrm{a}, 80 \mathrm{~b}}$ A. Bellora, ${ }^{80 \mathrm{a}}$ C. Biino, ${ }^{80 \mathrm{a}}$ A. Cappati ${ }^{80 \mathrm{a}, 80 \mathrm{~b}}$ N. Cartiglia, ${ }^{80 \mathrm{a}}$ S. Cometti, ${ }^{80 \mathrm{a}}$ M. Costa, ${ }^{80 \mathrm{a}, 80 \mathrm{~b}}$ R. Covarelli, ${ }^{80,80 b}$ N. Demaria, ${ }^{80 a}$ B. Kiani, ${ }^{80 a, 80 b}$ F. Legger, ${ }^{80 a}$ C. Mariotti, ${ }^{80 a}$ S. Maselli, ${ }^{80 a}$ E. Migliore,${ }^{80,80 b}$ V. Monaco, ${ }^{80 a, 80 b}$ E. Monteil, ${ }^{80 a, 80 b}$ M. Monteno, ${ }^{80 a}$ M. M. Obertino, ${ }^{80 a, 80 b}$ G. Ortona,${ }^{80 a, 80 b}$ L. Pacher ${ }^{80 a, 80 b}$ N. Pastrone, ${ }^{80 a}$ M. Pelliccioni, ${ }^{80 \mathrm{a}}$ G. L. Pinna Angioni, ${ }^{80 \mathrm{a}, 80 \mathrm{~b}}$ A. Romero, ${ }^{80 a, 80 \mathrm{~b}}$ M. Ruspa, ${ }^{80 \mathrm{a}, 80 \mathrm{c}}$ R. Salvatico, ${ }^{80 \mathrm{a}, 80 \mathrm{~b}}$ V. Sola, ${ }^{80 \mathrm{a}}$ A. Solano,${ }^{80 a, 80 b}$ D. Soldi, ${ }^{80 a, 80 b}$ A. Staiano, ${ }^{80 a}$ S. Belforte, ${ }^{81 a}$ V. Candelise, ${ }^{81 a, 81 b}$ M. Casarsa, ${ }^{81 a}$ F. Cossutti, ${ }^{81 a}$ A. Da Rold ${ }^{81 a, 81 b}$ G. Della Ricca, ${ }^{81 a, 81 b}$ F. Vazzoler, ${ }^{81 a, 81 b}$ A. Zanetti, ${ }^{81 a}$ B. Kim, ${ }^{82}$ D. H. Kim, ${ }^{82}$ G. N. Kim, ${ }^{82}$ J. Lee, ${ }^{82}$ S. W. Lee, ${ }^{82}$ C. S. Moon, ${ }^{82}$ Y. D. Oh ${ }^{82}$ S. I. Pak,${ }^{82}$ S. Sekmen, ${ }^{82}$ D. C. Son,${ }^{82}$ Y. C. Yang, ${ }^{82}$ H. Kim, ${ }^{83}$ D. H. Moon, ${ }^{83}$ G. Oh,${ }^{83}$ B. Francois, ${ }^{84}$ T. J. Kim, ${ }^{84}$ J. Park, ${ }^{84}$ S. Cho, ${ }^{85}$ S. Choi, ${ }^{85}$ Y. Go, ${ }^{85}$ S. Ha ${ }^{85}$ B. Hong,${ }^{85}$ K. Lee, ${ }^{85}$ K. S. Lee,${ }^{85}$ J. Lim, ${ }^{85}$ J. Park, ${ }^{85}$ S. K. Park,${ }^{85}$ Y. Roh, ${ }^{85}$ J. Yoo,${ }^{85}$ J. Goh,${ }^{86}$ H. S. Kim, ${ }^{87}$ J. Almond, ${ }^{88}$ J. H. Bhyun, ${ }^{88}$ J. Choi, ${ }^{88}$ S. Jeon, ${ }^{88}$ J. Kim, ${ }^{88}$ J. S. Kim,${ }^{88}$ H. Lee, ${ }^{88}$ K. Lee, ${ }^{88}$ S. Lee, ${ }^{88}$ K. Nam,${ }^{88}$ M. Oh ${ }^{88}$ S. B. Oh,${ }^{88}$ B. C. Radburn-Smith, ${ }^{88}$ U. K. Yang,${ }^{88}$ H. D. Yoo, ${ }^{88}$ I. Yoon, ${ }^{88}$ G. B. Yu ${ }^{88}$ D. Jeon,${ }^{89}$ H. Kim, ${ }^{89}$ J. H. Kim, ${ }^{89}$ J. S. H. Lee, ${ }^{89}$ I. C. Park, ${ }^{89}$ I. J Watson, ${ }^{89}$ Y. Choi, ${ }^{90}$ C. Hwang, ${ }^{90}$ Y. Jeong, ${ }^{90}$ J. Lee, ${ }^{90}$ Y. Lee, ${ }^{90}$ I. Yu, ${ }^{90}$ V. Veckalns,${ }^{91, i i}$ V. Dudenas, ${ }^{92}$ A. Juodagalvis, ${ }^{92}$ A. Rinkevicius, ${ }^{92}$ G. Tamulaitis, ${ }^{92}$ J. Vaitkus, ${ }^{92}$ Z. A. Ibrahim, ${ }^{93}$ F. Mohamad Idris, ${ }^{93, j j}$ W. A. T. Wan Abdullah, ${ }^{93}$ M. N. Yusli, ${ }^{93}$ Z. Zolkapli, ${ }^{93}$ J. F. Benitez, ${ }^{94}$ A. Castaneda Hernandez, ${ }^{94}$ J. A. Murillo Quijada, ${ }^{94}$ L. Valencia Palomo, ${ }^{94}$ H. Castilla-Valdez, ${ }^{95}$ E. De La Cruz-Burelo, ${ }^{95}$ I. Heredia-De La Cruz, ${ }^{95, k k}$ R. Lopez-Fernandez, ${ }^{95}$ A. Sanchez-Hernandez, ${ }^{95}$ S. Carrillo Moreno, ${ }^{96}$ C. Oropeza Barrera, ${ }^{96}$ M. Ramirez-Garcia, ${ }^{96}$ F. Vazquez Valencia, ${ }^{96}$ J. Eysermans, ${ }^{97}$ I. Pedraza,${ }^{97}$ H. A. Salazar Ibarguen, ${ }^{97}$ C. Uribe Estrada, ${ }^{97}$ A. Morelos Pineda, ${ }^{98}$ J. Mijuskovic, ${ }^{99, c}$ N. Raicevic, ${ }^{99}$ D. Krofcheck, ${ }^{100}$ S. Bheesette, ${ }^{101}$ P. H. Butler, ${ }^{101}$ A. Ahmad, ${ }^{102}$ M. Ahmad, ${ }^{102}$ Q. Hassan, ${ }^{102}$ H. R. Hoorani, ${ }^{102}$ W. A. Khan, ${ }^{102}$ M. A. Shah, ${ }^{102}$ M. Shoaib, ${ }^{102}$ M. Waqas, ${ }^{102}$ V. Avati, ${ }^{103}$ 
L. Grzanka, ${ }^{103}$ M. Malawski, ${ }^{103}$ H. Bialkowska, ${ }^{104}$ M. Bluj, ${ }^{104}$ B. Boimska, ${ }^{104}$ M. Górski, ${ }^{104}$ M. Kazana, ${ }^{104}$ M. Szleper, ${ }^{104}$ P. Zalewski, ${ }^{104}$ K. Bunkowski, ${ }^{105}$ A. Byszuk, ${ }^{105,11}$ K. Doroba, ${ }^{105}$ A. Kalinowski, ${ }^{105}$ M. Konecki, ${ }^{105}$ J. Krolikowski, ${ }^{105}$ M. Misiura, ${ }^{105}$ M. Olszewski, ${ }^{105}$ M. Walczak, ${ }^{105}$ M. Araujo, ${ }^{106}$ P. Bargassa, ${ }^{106}$ D. Bastos, ${ }^{106}$ A. Di Francesco, ${ }^{106}$ P. Faccioli, ${ }^{106}$ B. Galinhas, ${ }^{106}$ M. Gallinaro, ${ }^{106}$ J. Hollar, ${ }^{106}$ N. Leonardo, ${ }^{106}$ T. Niknejad, ${ }^{106}$ J. Seixas,${ }^{106}$ K. Shchelina, ${ }^{106}$ G. Strong, ${ }^{106}$ O. Toldaiev, ${ }^{106}$ J. Varela, ${ }^{106}$ S. Afanasiev, ${ }^{107}$ P. Bunin,,${ }^{107}$ M. Gavrilenko, ${ }^{107}$ I. Golutvin, ${ }^{107}$ I. Gorbunov, ${ }^{107}$ A. Kamenev, ${ }^{107}$ V. Karjavine, ${ }^{107}$ A. Lanev, ${ }^{107}$ A. Malakhov, ${ }^{107}$ V. Matveev, ${ }^{107, m m, n n}$ P. Moisenz, ${ }^{107}$ V. Palichik, ${ }^{107}$ V. Perelygin, ${ }^{107}$ M. Savina, ${ }^{107}$ S. Shmatov, ${ }^{107}$ S. Shulha,${ }^{107}$ N. Skatchkov, ${ }^{107}$ V. Smirnov, ${ }^{107}$ N. Voytishin,${ }^{107}$ A. Zarubin, ${ }^{107}$ L. Chtchipounov, ${ }^{108}$ V. Golovtcov, ${ }^{108}$ Y. Ivanov, ${ }^{108}$ V. Kim, ${ }^{108,00}$ E. Kuznetsova, ${ }^{108, p p}$ P. Levchenko, ${ }^{108}$ V. Murzin, ${ }^{108}$ V. Oreshkin, ${ }^{108}$ I. Smirnov, ${ }^{108}$ D. Sosnov, ${ }^{108}$ V. Sulimov, ${ }^{108}$ L. Uvarov, ${ }^{108}$ A. Vorobyev, ${ }^{108}$ Yu. Andreev, ${ }^{109}$ A. Dermenev, ${ }^{109}$ S. Gninenko, ${ }^{109}$ N. Golubev, ${ }^{109}$ A. Karneyeu, ${ }^{109}$ M. Kirsanov, ${ }^{109}$ N. Krasnikov, ${ }^{109}$ A. Pashenkov, ${ }^{109}$ D. Tlisov, ${ }^{109}$ A. Toropin, ${ }^{109}$ V. Epshteyn, ${ }^{110}$ V. Gavrilov, ${ }^{110}$ N. Lychkovskaya, ${ }^{110}$ A. Nikitenko, ${ }^{110, q q}$ V. Popov, ${ }^{110}$ I. Pozdnyakov, ${ }^{110}$ G. Safronov, ${ }_{110}$ A. Spiridonov, ${ }^{110}$ A. Stepennov, ${ }^{110}$ M. Toms, ${ }^{110}$ E. Vlasov, ${ }^{110}$ A. Zhokin, ${ }^{110}$ T. Aushev, ${ }^{111}$ M. Chadeeva, ${ }^{112, r r}$ P. Parygin, ${ }^{112}$ D. Philippov, ${ }^{112}$ E. Popova, ${ }^{112}$ V. Rusinov, ${ }^{112}$ V. Andreev, ${ }^{113}$ M. Azarkin, ${ }^{113}$ I. Dremin, ${ }^{113}$ M. Kirakosyan, ${ }^{113}$ A. Terkulov, ${ }^{113}$ A. Baskakov, ${ }^{114}$ A. Belyaev, ${ }^{114}$ E. Boos, ${ }^{114}$ V. Bunichev, ${ }^{114}$ M. Dubinin, ${ }^{114, s s}$ L. Dudko, ${ }^{114}$ V. Klyukhin, ${ }^{114}$ N. Korneeva, ${ }^{114}$ I. Lokhtin, ${ }^{114}$ S. Obraztsov, ${ }^{114}$ M. Perfilov, ${ }^{114}$ V. Savrin, ${ }^{114}$ P. Volkov, ${ }^{114}$ A. Barnyakov, ${ }^{115, t t}$ V. Blinov, ${ }^{15, t t}$ T. Dimova, ${ }^{115, \mathrm{tt}}$ L. Kardapoltsev, ${ }^{115, \mathrm{tt}}$ Y. Skovpen, ${ }^{115, \mathrm{tt}}$ I. Azhgirey, ${ }^{116}$ I. Bayshev, ${ }^{116}$ S. Bitioukov, ${ }^{116}$ V. Kachanov, ${ }^{116}$ D. Konstantinov, ${ }^{116}$ P. Mandrik, ${ }^{116}$ V. Petrov ${ }^{116}$ R. Ryutin, ${ }^{116}$ S. Slabospitskii, ${ }^{116}$ A. Sobol, ${ }^{116}$ S. Troshin, ${ }^{116}$ N. Tyurin, ${ }^{116}$ A. Uzunian, ${ }^{116}$ A. Volkov, ${ }^{116}$ A. Babaev, ${ }^{117}$ A. Iuzhakov, ${ }^{117}$ V. Okhotnikov, ${ }^{117}$ V. Borchsh, ${ }^{118}$ V. Ivanchenko, ${ }^{118}$ E. Tcherniaev, ${ }^{118}$ P. Adzic, ${ }^{119, \text { uu }}$ P. Cirkovic, ${ }^{119}$ M. Dordevic, ${ }^{119}$ P. Milenovic, ${ }^{119}$ J. Milosevic,${ }^{119}$ M. Stojanovic, ${ }^{119}$ M. Aguilar-Benitez, ${ }^{120}$ J. Alcaraz Maestre, ${ }^{120}$ A. Álvarez Fernández, ${ }^{120}$ I. Bachiller, ${ }^{120}$ M. Barrio Luna, ${ }^{120}$ J. A. Brochero Cifuentes, ${ }^{120}$ C. A. Carrillo Montoya, ${ }^{120}$ M. Cepeda, ${ }^{120}$ M. Cerrada, ${ }^{120}$ N. Colino, ${ }^{120}$ B. De La Cruz, ${ }^{120}$ A. Delgado Peris, ${ }^{120}$ C. Fernandez Bedoya,${ }^{120}$ J. P. Fernández Ramos, ${ }^{120}$ J. Flix, ${ }^{120}$ M. C. Fouz, ${ }^{120}$ O. Gonzalez Lopez, ${ }^{120}$ S. Goy Lopez, ${ }^{120}$ J. M. Hernandez, ${ }^{120}$ M. I. Josa, ${ }^{120}$ D. Moran, ${ }^{120}$ Á. Navarro Tobar, ${ }^{120}$ A. Pérez-Calero Yzquierdo, ${ }^{120}$ J. Puerta Pelayo, ${ }^{120}$ I. Redondo, ${ }^{120}$ L. Romero, ${ }^{120}$ S. Sánchez Navas, ${ }^{120}$ M. S. Soares, ${ }^{120}$ A. Triossi, ${ }^{120}$ C. Willmott, ${ }^{120}$ C. Albajar, ${ }^{121}$ J. F. de Trocóniz, ${ }^{121}$ R. Reyes-Almanza, ${ }^{121}$ B. Alvarez Gonzalez, ${ }^{122}$ J. Cuevas, ${ }^{122}$ C. Erice, ${ }^{122}$ J. Fernandez Menendez, ${ }^{122}$ S. Folgueras, ${ }^{122}$ I. Gonzalez Caballero, ${ }^{122}$ J. R. González Fernández, ${ }^{122}$ E. Palencia Cortezon, ${ }^{122}$ V. Rodríguez Bouza, ${ }^{122}$ S. Sanchez Cruz, ${ }^{122}$ I. J. Cabrillo, ${ }^{123}$ A. Calderon, ${ }^{123}$ B. Chazin Quero, ${ }^{123}$ J. Duarte Campderros, ${ }^{123}$ M. Fernandez, ${ }^{123}$ P. J. Fernández Manteca, ${ }^{123}$ A. García Alonso, ${ }^{123}$ G. Gomez, ${ }^{123}$ C. Martinez Rivero, ${ }^{123}$ P. Martinez Ruiz del Arbol, ${ }^{123}$ F. Matorras, ${ }^{123}$ J. Piedra Gomez, ${ }^{123}$ C. Prieels, ${ }^{123}$ T. Rodrigo, ${ }^{123}$ A. Ruiz-Jimeno, ${ }^{123}$ L. Russo, ${ }^{123, v v}$ L. Scodellaro, ${ }^{123}$ I. Vila, ${ }^{123}$ J. M. Vizan Garcia, ${ }^{123}$ K. Malagalage, ${ }^{124}$ W. G. D. Dharmaratna, ${ }^{125}$ N. Wickramage, ${ }^{125}$ D. Abbaneo, ${ }^{126}$ B. Akgun, ${ }^{126}$ E. Auffray, ${ }^{126}$ G. Auzinger, ${ }^{126}$ J. Baechler, ${ }^{126}$ P. Baillon, ${ }^{126}$ A. H. Ball, ${ }^{126}$ D. Barney, ${ }^{126}$ J. Bendavid, ${ }^{126}$ M. Bianco, ${ }^{126}$ A. Bocci, ${ }^{126}$ P. Bortignon, ${ }^{126}$ E. Bossini, ${ }^{126}$ C. Botta, ${ }^{126}$ E. Brondolin, ${ }^{126}$

T. Camporesi, ${ }^{126}$ A. Caratelli, ${ }^{126}$ G. Cerminara, ${ }^{126}$ E. Chapon, ${ }^{126}$ G. Cucciati, ${ }^{126}$ D. d'Enterria, ${ }^{126}$ A. Dabrowski, ${ }^{126}$ N. Daci, ${ }^{126}$ V. Daponte, ${ }^{126}$ A. David, ${ }^{126}$ O. Davignon, ${ }^{126}$ A. De Roeck, ${ }^{126}$ M. Deile, ${ }^{126}$ M. Dobson, ${ }^{126}$ M. Dünser, ${ }^{126}$ N. Dupont, ${ }^{126}$ A. Elliott-Peisert, ${ }^{126}$ N. Emriskova, ${ }^{126}$ F. Fallavollita, ${ }^{126, w w}$ D. Fasanella, ${ }^{126}$ S. Fiorendi, ${ }^{126}$ G. Franzoni, ${ }^{126}$ J. Fulcher, ${ }^{126}$ W. Funk, ${ }^{126}$ S. Giani, ${ }^{126}$ D. Gigi, ${ }^{126}$ A. Gilbert, ${ }^{126}$ K. Gill, ${ }^{126}$ F. Glege, ${ }^{126}$ L. Gouskos, ${ }^{126}$ M. Gruchala, ${ }^{126}$ M. Guilbaud, ${ }^{126}$ D. Gulhan, ${ }^{126}$ J. Hegeman, ${ }^{126}$ C. Heidegger, ${ }^{126}$ Y. Iiyama, ${ }^{126}$ V. Innocente, ${ }^{126}$ T. James, ${ }^{126}$ P. Janot, ${ }^{126}$

O. Karacheban, ${ }^{126, v}$ J. Kaspar, ${ }^{126}$ J. Kieseler, ${ }^{126}$ M. Krammer, ${ }^{126, b}$ N. Kratochwil, ${ }^{126}$ C. Lange, ${ }^{126}$ P. Lecoq, ${ }^{126}$

C. Lourenço, ${ }^{126}$ L. Malgeri, ${ }^{126}$ M. Mannelli, ${ }^{126}$ A. Massironi, ${ }^{126}$ F. Meijers, ${ }^{126}$ J. A. Merlin, ${ }^{126}$ S. Mersi, ${ }^{126}$ E. Meschi, ${ }^{126}$

F. Moortgat, ${ }^{126}$ M. Mulders, ${ }^{126}$ J. Ngadiuba ${ }^{126}$ J. Niedziela, ${ }^{126}$ S. Nourbakhsh, ${ }^{126}$ S. Orfanelli, ${ }^{126}$ L. Orsini, ${ }^{126}$ F. Pantaleo, ${ }^{126, \mathrm{~s}}$ L. Pape, ${ }^{126}$ E. Perez, ${ }^{126}$ M. Peruzzi, ${ }^{126}$ A. Petrilli, ${ }^{126}$ G. Petrucciani, ${ }^{126}$ A. Pfeiffer, ${ }^{126}$ M. Pierini, ${ }^{126}$ F. M. Pitters, ${ }^{126}$ D. Rabady, ${ }^{126}$ A. Racz, ${ }^{126}$ M. Rieger, ${ }^{126}$ M. Rovere, ${ }^{126}$ H. Sakulin, ${ }^{126}$ C. Schäfer, ${ }^{126}$ C. Schwick, ${ }^{126}$ M. Selvaggi, ${ }^{126}$ A. Sharma, ${ }^{126}$ P. Silva, ${ }^{126}$ W. Snoeys, ${ }^{126}$ P. Sphicas, ${ }^{126, x x}$ J. Steggemann, ${ }^{126}$ S. Summers, ${ }^{126}$ V. R. Tavolaro, ${ }^{126}$ D. Treille, ${ }^{126}$ A. Tsirou, ${ }^{126}$ G. P. Van Onsem, ${ }^{126}$ A. Vartak, ${ }^{126}$ M. Verzetti, ${ }^{126}$ W. D. Zeuner, ${ }^{126}$ L. Caminada, ${ }^{127, y y}$ K. Deiters, ${ }^{127}$ W. Erdmann, ${ }^{127}$ R. Horisberger,${ }^{127}$ Q. Ingram,${ }^{127}$ H. C. Kaestli, ${ }^{127}$ D. Kotlinski, ${ }^{127}$ U. Langenegger, ${ }^{127}$ T. Rohe, ${ }^{127}$ S. A. Wiederkehr, ${ }^{127}$ M. Backhaus, ${ }^{128}$ P. Berger, ${ }^{128}$ N. Chernyavskaya, ${ }^{128}$ G. Dissertori, ${ }^{128}$ M. Dittmar, ${ }^{128}$ M. Donegà, ${ }^{128}$ C. Dorfer, ${ }^{128}$ T. A. Gómez Espinosa, ${ }^{128}$ C. Grab, ${ }^{128}$ D. Hits, ${ }^{128}$ W. Lustermann, ${ }^{128}$ R. A. Manzoni, ${ }^{128}$ M. T. Meinhard, ${ }^{128}$ F. Micheli, ${ }^{128}$ P. Musella, ${ }^{128}$ F. Nessi-Tedaldi, ${ }^{128}$ F. Pauss, ${ }^{128}$ G. Perrin, ${ }^{128}$ L. Perrozzi, ${ }^{128}$ S. Pigazzini, ${ }^{128}$ M. G. Ratti, ${ }^{128}$ M. Reichmann, ${ }^{128}$ C. Reissel,${ }^{128}$ T. Reitenspiess, ${ }^{128}$ D. Ruini, ${ }^{128}$ 
D. A. Sanz Becerra, ${ }^{128}$ M. Schönenberger, ${ }^{128}$ L. Shchutska, ${ }^{128}$ M. L. Vesterbacka Olsson, ${ }^{128}$ R. Wallny, ${ }^{128}$ D. H. Zhu, ${ }^{128}$ T. K. Aarrestad, ${ }^{129}$ C. Amsler, ${ }^{129, z z}$ D. Brzhechko, ${ }^{129}$ M. F. Canelli, ${ }^{129}$ A. De Cosa, ${ }^{129}$ R. Del Burgo, ${ }^{129}$ B. Kilminster, ${ }^{129}$ S. Leontsinis, ${ }^{129}$ V. M. Mikuni, ${ }^{129}$ I. Neutelings, ${ }^{129}$ G. Rauco, ${ }^{129}$ P. Robmann, ${ }^{129}$ K. Schweiger, ${ }^{129}$ C. Seitz, ${ }^{129}$ Y. Takahashi, ${ }^{129}$ S. Wertz, ${ }^{129}$ A. Zucchetta, ${ }^{129}$ T. H. Doan, ${ }^{130}$ C. M. Kuo, ${ }^{130}$ W. Lin, ${ }^{130}$ A. Roy, ${ }^{130}$ S. S. Yu, ${ }^{130}$ P. Chang, ${ }^{131}$ Y. Chao, ${ }^{131}$ K. F. Chen, ${ }^{131}$ P. H. Chen, ${ }^{131}$ W.-S. Hou, ${ }^{131}$ Y. y. Li, ${ }^{131}$ R.-S. Lu,${ }^{131}$ E. Paganis, ${ }^{131}$ A. Psallidas, ${ }^{131}$ A. Steen, ${ }^{131}$ B. Asavapibhop, ${ }^{132}$ C. Asawatangtrakuldee ${ }^{132}$ N. Srimanobhas, ${ }^{132}$ N. Suwonjandee, ${ }^{132}$ A. Bat,${ }^{133}$ F. Boran, ${ }^{133}$

A. Celik, ${ }^{133 \text {,aaa }}$ S. Cerci, ${ }^{133, \text { bbb }}$ S. Damarseckin, ${ }^{133, \text { ccc }}$ Z. S. Demiroglu, ${ }^{133}$ F. Dolek, ${ }^{133}$ C. Dozen, ${ }^{133, \text { ddd }}$ I. Dumanoglu, ${ }^{133}$ G. Gokbulut, ${ }^{133}$ Emine Gurpinar Guler, ${ }^{133, \text { eee }}$ Y. Guler, ${ }^{133}$ I. Hos, ${ }^{133, f f f}$ C. Isik, ${ }^{133}$ E. E. Kangal,,${ }^{133, \text { ggg }}$ O. Kara, ${ }^{133}$ U. Kiminsu, ${ }^{133}$ G. Onengut, ${ }^{133}$ K. Ozdemir, ${ }^{133, \text { hhh }}$ S. Ozturk, ${ }^{133, \text { iii }}$ A. Polatoz, ${ }^{133}$ A. E. Simsek, ${ }^{133}$ D. Sunar Cerci, ${ }^{133, \text { bbb }}$ U. G. Tok, ${ }^{133}$ S. Turkcapar, ${ }^{133}$ I. S. Zorbakir, ${ }^{133}$ C. Zorbilmez, ${ }^{133}$ B. Isildak, ${ }^{134, j j j}$ G. Karapinar, ${ }^{134, k k k}$ M. Yalvac, ${ }^{134}$ I. O. Atakisi, ${ }^{135}$ E. Gülmez, ${ }^{135}$ M. Kaya, ${ }^{135,111}$ O. Kaya, ${ }^{135, \mathrm{mmm}}$ Ö. Özçelik, ${ }^{135}$ S. Tekten, ${ }^{135}$ E. A. Yetkin, ${ }^{135, \text { nnn }}$ A. Cakir, ${ }^{136}$ K. Cankocak, ${ }^{136}$ Y. Komurcu, ${ }^{136}$ S. Sen ${ }^{136,000}$ B. Kaynak, ${ }^{137}$ S. Ozkorucuklu,${ }^{137}$ B. Grynyov, ${ }^{138}$ L. Levchuk, ${ }^{139}$ E. Bhal, ${ }^{140}$ S. Bologna, ${ }^{140}$ J. J. Brooke, ${ }^{140}$ D. Burns, ${ }^{140, p p p}$ E. Clement, ${ }^{140}$ D. Cussans, ${ }^{140}$ H. Flacher, ${ }^{140}$ J. Goldstein, ${ }^{140}$ G. P. Heath, ${ }^{140}$ H. F. Heath, ${ }^{140}$ L. Kreczko, ${ }^{140}$ B. Krikler, ${ }^{140}$ S. Paramesvaran, ${ }^{140}$ B. Penning, ${ }^{140}$ T. Sakuma, ${ }^{140}$ S. Seif El Nasr-Storey, ${ }^{140}$ V. J. Smith, ${ }^{140}$ J. Taylor, ${ }^{140}$ A. Titterton, ${ }^{140}$ K. W. Bell, ${ }^{141}$ A. Belyaev, ${ }^{141, q q q}$ C. Brew, ${ }^{141}$ R. M. Brown, ${ }^{141}$

D. J. A. Cockerill, ${ }^{141}$ J. A. Coughlan, ${ }^{141}$ K. Harder,${ }^{141}$ S. Harper, ${ }^{141}$ J. Linacre, ${ }^{141}$ K. Manolopoulos, ${ }^{141}$ D. M. Newbold, ${ }^{141}$ E. Olaiya, ${ }^{141}$ D. Petyt, ${ }^{141}$ T. Reis, ${ }^{141}$ T. Schuh, ${ }^{141}$ C. H. Shepherd-Themistocleous, ${ }^{141}$ A. Thea, ${ }^{141}$ I. R. Tomalin, ${ }^{141}$ T. Williams, ${ }^{141}$ W. J. Womersley, ${ }^{141}$ R. Bainbridge, ${ }^{142}$ P. Bloch, ${ }^{142}$ J. Borg, ${ }^{142}$ S. Breeze,${ }^{142}$ O. Buchmuller, ${ }^{142}$ A. Bundock, ${ }^{142}$ Gurpreet Singh CHAHAL, ${ }^{142, \text { rrr }}$ D. Colling, ${ }^{142}$ P. Dauncey, ${ }^{142}$ G. Davies, ${ }^{142}$ M. Della Negra, ${ }^{142}$ R. Di Maria, ${ }^{142}$ P. Everaerts, ${ }^{142}$ G. Hall, ${ }^{142}$ G. Iles, ${ }^{142}$ M. Komm, ${ }^{142}$ C. Laner, ${ }^{142}$ L. Lyons, ${ }^{142}$ A.-M. Magnan, ${ }^{142}$ S. Malik, ${ }^{142}$ A. Martelli, ${ }^{142}$ V. Milosevic, ${ }^{142}$ A. Morton, ${ }^{142}$ J. Nash,${ }^{142, \text { sss }}$ V. Palladino, ${ }^{142}$ M. Pesaresi, ${ }^{142}$ D. M. Raymond, ${ }^{142}$ A. Richards, ${ }^{142}$ A. Rose, ${ }^{142}$ E. Scott, ${ }^{142}$ C. Seez, ${ }^{142}$ A. Shtipliyski, ${ }^{142}$ M. Stoye, ${ }^{142}$ T. Strebler, ${ }^{142}$ A. Tapper, ${ }^{142}$ K. Uchida, ${ }^{142}$

T. Virdee ${ }^{142, \mathrm{~s}}$ N. Wardle, ${ }^{142}$ D. Winterbottom, ${ }^{142}$ J. Wright, ${ }^{142}$ A. G. Zecchinelli, ${ }^{142}$ S. C. Zenz, ${ }^{142}$ J. E. Cole, ${ }^{143}$ P. R. Hobson, ${ }^{143}$ A. Khan, ${ }^{143}$ P. Kyberd,${ }^{143}$ C. K. Mackay, ${ }^{143}$ I. D. Reid, ${ }^{143}$ L. Teodorescu, ${ }^{143}$ S. Zahid, ${ }^{143}$ K. Call, ${ }^{144}$ B. Caraway, ${ }^{144}$ J. Dittmann, ${ }^{144}$ K. Hatakeyama, ${ }^{144}$ C. Madrid ${ }^{144}$ B. McMaster, ${ }^{144}$ N. Pastika,${ }^{144}$ C. Smith ${ }^{144}$ R. Bartek,${ }^{145}$ A. Dominguez, ${ }^{145}$ R. Uniyal, ${ }^{145}$ A. M. Vargas Hernandez, ${ }^{145}$ A. Buccilli, ${ }^{146}$ S. I. Cooper, ${ }^{146}$ C. Henderson, ${ }^{146}$ P. Rumerio, ${ }^{146}$ C. West,${ }^{146}$ A. Albert,${ }^{147}$ D. Arcaro, ${ }^{147}$ Z. Demiragli, ${ }^{147}$ D. Gastler,${ }^{147}$ C. Richardson, ${ }^{147}$ J. Rohlf, ${ }^{147}$ D. Sperka, ${ }^{147}$ I. Suarez ${ }^{147}$ L. Sulak,${ }^{147}$ D. Zou ${ }^{147}$ G. Benelli, ${ }^{148}$ B. Burkle, ${ }^{148}$ X. Coubez,${ }^{148, t}$ D. Cutts, ${ }^{148}$ Y. t. Duh,${ }^{148}$ M. Hadley, ${ }^{148}$ U. Heintz, ${ }^{148}$ J. M. Hogan, ${ }^{148, \text { ttt }}$ K. H. M. Kwok, ${ }^{148}$ E. Laird, ${ }^{148}$ G. Landsberg, ${ }^{148}$ K. T. Lau, ${ }^{148}$ J. Lee, ${ }^{148}$ Z. Mao, ${ }^{148}$ M. Narain, ${ }^{148}$ S. Sagir, ${ }^{148, \text { uuu }}$ R. Syarif, ${ }^{148}$ E. Usai, ${ }^{148}$ D. Yu, ${ }^{148}$ W. Zhang, ${ }^{148}$ R. Band, ${ }^{149}$ C. Brainerd, ${ }^{149}$ R. Breedon, ${ }^{149}$ M. Calderon De La Barca Sanchez, ${ }^{149}$ M. Chertok, ${ }^{149}$ J. Conway, ${ }^{149}$ R. Conway, ${ }^{149}$ P. T. Cox,${ }^{149}$ R. Erbacher, ${ }^{149}$ C. Flores, ${ }^{149}$ G. Funk ${ }^{149}$ F. Jensen, ${ }^{149}$ W. Ko, ${ }^{149}$ O. Kukral,${ }^{149}$ R. Lander ${ }^{149}$ M. Mulhearn, ${ }^{149}$ D. Pellett, ${ }^{149}$ J. Pilot, ${ }^{149}$ M. Shi, ${ }^{149}$ D. Taylor, ${ }^{149}$ K. Tos, ${ }^{149}$ M. Tripathi, ${ }^{149}$ Z. Wang, ${ }^{149}$ F. Zhang, ${ }^{149}$ M. Bachtis, ${ }^{150}$ C. Bravo,${ }^{150}$ R. Cousins,${ }^{150}$ A. Dasgupta, ${ }^{150}$ A. Florent, ${ }^{150}$ J. Hauser, ${ }^{150}$ M. Ignatenko, ${ }^{150}$ N. Mccoll, ${ }^{150}$ W. A. Nash, ${ }^{150}$ S. Regnard, ${ }^{150}$ D. Saltzberg, ${ }^{150}$ C. Schnaible, ${ }^{150}$ B. Stone, ${ }^{150}$ V. Valuev, ${ }^{150}$ K. Burt, ${ }^{151}$ Y. Chen, ${ }^{151}$ R. Clare, ${ }^{151}$ J. W. Gary, ${ }^{151}$ S. M. A. Ghiasi Shirazi, ${ }^{151}$ G. Hanson, ${ }^{151}$ G. Karapostoli, ${ }^{151}$ E. Kennedy, ${ }^{151}$ O. R. Long, ${ }^{151}$ M. Olmedo Negrete, ${ }^{151}$ M. I. Paneva, ${ }^{151}$ W. Si, ${ }^{151}$ L. Wang, ${ }^{151}$ S. Wimpenny, ${ }^{151}$ B. R. Yates, ${ }^{151}$ Y. Zhang, ${ }^{151}$ J. G. Branson, ${ }^{152}$ P. Chang, ${ }^{152}$ S. Cittolin, ${ }^{152}$ S. Cooperstein, ${ }^{152}$ N. Deelen, ${ }^{152}$ M. Derdzinski, ${ }^{152}$ R. Gerosa, ${ }^{152}$ D. Gilbert, ${ }^{152}$ B. Hashemi ${ }^{152}$ D. Klein, ${ }^{152}$ V. Krutelyov, ${ }^{152}$ J. Letts, ${ }^{152}$ M. Masciovecchio, ${ }^{152}$

S. May, ${ }^{152}$ S. Padhi, ${ }^{152}$ M. Pieri, ${ }^{152}$ V. Sharma,${ }^{152}$ M. Tadel, ${ }^{152}$ F. Würthwein, ${ }^{152}$ A. Yagil,${ }^{152}$ G. Zevi Della Porta, ${ }^{152}$ N. Amin, ${ }^{153}$ R. Bhandari, ${ }^{153}$ C. Campagnari, ${ }^{153}$ M. Citron, ${ }^{153}$ V. Dutta, ${ }^{153}$ M. Franco Sevilla, ${ }^{153}$ J. Incandela, ${ }^{153}$ B. Marsh, ${ }^{153}$ H. Mei, ${ }^{153}$ A. Ovcharova, ${ }^{153}$ H. Qu, ${ }^{153}$ J. Richman, ${ }^{153}$ U. Sarica, ${ }^{153}$ D. Stuart, ${ }^{153}$ S. Wang, ${ }^{153}$ D. Anderson, ${ }^{154}$ A. Bornheim, ${ }^{154}$ O. Cerri, ${ }^{154}$ I. Dutta, ${ }^{154}$ J. M. Lawhorn, ${ }^{154}$ N. Lu,${ }^{154}$ J. Mao, ${ }^{154}$ H. B. Newman, ${ }^{154}$ T. Q. Nguyen, ${ }^{154}$ J. Pata, ${ }^{154}$ M. Spiropulu, ${ }^{154}$ J. R. Vlimant, ${ }^{154}$ S. Xie, ${ }^{154}$ Z. Zhang,,${ }^{154}$ R. Y. Zhu, ${ }^{154}$ M. B. Andrews, ${ }^{155}$ T. Ferguson, ${ }^{155}$ T. Mudholkar, ${ }^{155}$ M. Paulini, ${ }^{155}$ M. Sun,${ }^{155}$ I. Vorobiev, ${ }^{155}$ M. Weinberg, ${ }^{155}$ J. P. Cumalat, ${ }^{156}$ W. T. Ford, ${ }^{156}$ E. MacDonald, ${ }^{156}$ T. Mulholland, ${ }^{156}$ R. Patel, ${ }^{156}$ A. Perloff, ${ }^{156}$ K. Stenson, ${ }^{156}$ K. A. Ulmer, ${ }^{156}$ S. R. Wagner, ${ }^{156}$ J. Alexander, ${ }^{157}$ Y. Cheng, ${ }^{157}$ J. Chu, ${ }^{157}$ A. Datta, ${ }^{157}$ A. Frankenthal,${ }^{157}$ K. Mcdermott, ${ }^{157}$ J. R. Patterson, ${ }^{157}$ D. Quach, ${ }^{157}$ A. Ryd, ${ }^{157}$ S. M. Tan, ${ }^{157}$ Z. Tao, ${ }^{157}$ J. Thom, ${ }^{157}$ P. Wittich, ${ }^{157}$ M. Zientek, ${ }^{157}$ S. Abdullin, ${ }^{158}$ M. Albrow, ${ }^{158}$ M. Alyari, ${ }^{158}$ G. Apollinari, ${ }^{158}$ A. Apresyan, ${ }^{158}$ A. Apyan, ${ }^{158}$ S. Banerjee, ${ }^{158}$ L. A. T. Bauerdick, ${ }^{158}$ A. Beretvas, ${ }^{158}$ D. Berry, ${ }^{158}$ J. Berryhill, ${ }^{158}$ P. C. Bhat,${ }^{158}$ K. Burkett, ${ }^{158}$ J. N. Butler, ${ }^{158}$ A. Canepa, ${ }^{158}$ G. B. Cerati, ${ }^{158}$ H. W. K. Cheung, ${ }^{158}$ F. Chlebana, ${ }^{158}$ M. Cremonesi, ${ }^{158}$ 
J. Duarte, ${ }^{158}$ V. D. Elvira, ${ }^{158}$ J. Freeman, ${ }^{158}$ Z. Gecse, ${ }^{158}$ E. Gottschalk, ${ }^{158}$ L. Gray, ${ }^{158}$ D. Green, ${ }^{158}$ S. Grünendahl, ${ }^{158}$ O. Gutsche, ${ }^{158}$ Allison Reinsvold Hall, ${ }^{158}$ J. Hanlon, ${ }^{158}$ R. M. Harris ${ }^{158}$ S. Hasegawa ${ }^{158}$ R. Heller, ${ }^{158}$ J. Hirschauer, ${ }^{158}$ B. Jayatilaka, ${ }^{158}$ S. Jindariani, ${ }^{158}$ M. Johnson, ${ }^{158}$ U. Joshi, ${ }^{158}$ T. Klijnsma, ${ }^{158}$ B. Klima, ${ }^{158}$ M. J. Kortelainen, ${ }^{158}$ B. Kreis, ${ }^{158}$ S. Lammel, ${ }^{158}$ J. Lewis, ${ }^{158}$ D. Lincoln, ${ }^{158}$ R. Lipton, ${ }^{158}$ M. Liu, ${ }^{158}$ T. Liu, ${ }^{158}$ J. Lykken, ${ }^{158}$ K. Maeshima, ${ }^{158}$ J. M. Marraffino, ${ }^{158}$ D. Mason, ${ }^{158}$ P. McBride, ${ }^{158}$ P. Merkel, ${ }^{158}$ S. Mrenna, ${ }^{158}$ S. Nahn, ${ }^{158}$ V. O’ Dell, ${ }^{158}$ V. Papadimitriou, ${ }^{158}$ K. Pedro, ${ }^{158}$ C. Pena, ${ }^{158}$ G. Rakness, ${ }^{158}$ F. Ravera,${ }^{158}$ L. Ristori, ${ }^{158}$ B. Schneider, ${ }^{158}$ E. Sexton-Kennedy, ${ }^{158}$ N. Smith, ${ }^{158}$ A. Soha, ${ }^{158}$ W. J. Spalding, ${ }^{158}$ L. Spiegel, ${ }^{158}$ S. Stoynev, ${ }^{158}$ J. Strait ${ }^{158}$ N. Strobbe,${ }^{158}$ L. Taylor, ${ }^{158}$ S. Tkaczyk, ${ }^{158}$ N. V. Tran, ${ }^{158}$ L. Uplegger, ${ }^{158}$ E. W. Vaandering, ${ }^{158}$ C. Vernieri, ${ }^{158}$ R. Vidal, ${ }^{158}$ M. Wang, ${ }^{158}$ H. A. Weber, ${ }^{158}$ D. Acosta, ${ }^{159}$ P. Avery, ${ }^{159}$ D. Bourilkov, ${ }^{159}$ A. Brinkerhoff,${ }^{159}$ L. Cadamuro, ${ }^{159}$ A. Carnes, ${ }^{159}$ V. Cherepanov, ${ }^{159}$ F. Errico,${ }^{159}$ R. D. Field,${ }^{159}$ S. V. Gleyzer, ${ }^{159}$ B. M. Joshi, ${ }^{159}$ M. Kim, ${ }^{159}$ J. Konigsberg, ${ }^{159}$ A. Korytov, ${ }^{159}$ K. H. Lo, ${ }^{159}$ P. Ma, ${ }^{159}$ K. Matchev, ${ }^{159}$ N. Menendez, ${ }^{159}$ G. Mitselmakher, ${ }^{159}$ D. Rosenzweig, ${ }^{159}$ K. Shi, ${ }^{159}$ J. Wang, ${ }^{159}$ S. Wang, ${ }^{159}$ X. Zuo, ${ }^{159}$ Y. R. Joshi, ${ }^{160}$ T. Adams,${ }^{161}$ A. Askew, ${ }^{161}$ S. Hagopian,${ }^{161}$ V. Hagopian, ${ }^{161}$ K. F. Johnson, ${ }^{161}$ R. Khurana, ${ }^{161}$ T. Kolberg, ${ }^{161}$ G. Martinez, ${ }^{161}$ T. Perry, ${ }^{161}$ H. Prosper, ${ }^{161}$ C. Schiber, ${ }^{161}$ R. Yohay, ${ }^{161}$ J. Zhang, ${ }^{161}$ M. M. Baarmand, ${ }^{162}$ M. Hohlmann, ${ }^{162}$ D. Noonan, ${ }^{162}$ M. Rahmani, ${ }^{162}$ M. Saunders, ${ }^{162}$ F. Yumiceva, ${ }^{162}$ M. R. Adams, ${ }^{163}$ L. Apanasevich, ${ }^{163}$ R. R. Betts, ${ }^{163}$ R. Cavanaugh, ${ }^{163}$ X. Chen, ${ }^{163}$ S. Dittmer, ${ }^{163}$ O. Evdokimov, ${ }^{163}$ C. E. Gerber, ${ }^{163}$ D. A. Hangal, ${ }^{163}$ D. J. Hofman, ${ }^{163}$ K. Jung, ${ }^{163}$ C. Mills, ${ }^{163}$ T. Roy, ${ }_{163}$ M. B. Tonjes, ${ }^{163}$ N. Varelas, ${ }^{163}$ J. Viinikainen, ${ }^{163}$ H. Wang, ${ }^{163}$ X. Wang, ${ }^{163} \mathrm{Z}$. Wu, ${ }^{163}$ M. Alhusseini, ${ }^{164}$ B. Bilki, ${ }^{164, \text { eee }}$ W. Clarida ${ }^{164}$ K. Dilsiz, ${ }^{164, v v v}$ S. Durgut, ${ }^{164}$ R. P. Gandrajula, ${ }^{164}$ M. Haytmyradov, ${ }^{164}$ V. Khristenko, ${ }^{164}$ O. K. Köseyan, ${ }^{164}$ J.-P. Merlo, ${ }^{164}$ A. Mestvirishvili, ${ }^{164, w w w}$ A. Moeller, ${ }^{164}$ J. Nachtman, ${ }^{164}$ H. Ogul, ${ }^{164, x x x}$ Y. Onel, ${ }^{164}$ F. Ozok, ${ }^{164, y y y}$ A. Penzo, ${ }^{164}$ C. Snyder,${ }^{164}$ E. Tiras, ${ }^{164}$ J. Wetzel,${ }^{164}$ B. Blumenfeld, ${ }^{165}$ A. Cocoros, ${ }^{165}$ N. Eminizer, ${ }^{165}$ A. V. Gritsan, ${ }^{165}$ W. T. Hung, ${ }^{165}$ S. Kyriacou, ${ }^{165}$ P. Maksimovic, ${ }^{165}$ J. Roskes, ${ }^{165}$ M. Swartz, ${ }^{165}$ C. Baldenegro Barrera, ${ }^{166}$ P. Baringer, ${ }^{166}$ A. Bean, ${ }^{166}$ S. Boren, ${ }^{166}$ J. Bowen, ${ }^{166}$ A. Bylinkin, ${ }^{166}$ T. Isidori, ${ }^{166}$ S. Khalil, ${ }^{166}$ J. King, ${ }^{166}$ G. Krintiras, ${ }^{166}$ A. Kropivnitskaya, ${ }^{166}$ C. Lindsey, ${ }^{166}$ D. Majumder, ${ }^{166}$ W. Mcbrayer,${ }^{166}$ N. Minafra ${ }^{166}$ M. Murray, ${ }^{166}$ C. Rogan, ${ }^{166}$ C. Royon, ${ }^{166}$ S. Sanders, ${ }^{166}$ E. Schmitz, ${ }^{166}$ J. D. Tapia Takaki, ${ }^{166}$ Q. Wang, ${ }^{166}$ J. Williams, ${ }^{166}$ G. Wilson, ${ }^{166}$ S. Duric, ${ }^{167}$ A. Ivanov, ${ }^{167}$ K. Kaadze, ${ }^{167}$ D. Kim, ${ }^{167}$ Y. Maravin, ${ }^{167}$ D. R. Mendis, ${ }^{167}$ T. Mitchell, ${ }^{167}$ A. Modak, ${ }^{167}$ A. Mohammadi, ${ }^{167}$ F. Rebassoo, ${ }^{168}$ D. Wright, ${ }^{168}$ A. Baden, ${ }^{169}$ O. Baron, ${ }^{169}$ A. Belloni, ${ }^{169}$ S. C. Eno, ${ }^{169}$ Y. Feng, ${ }^{169}$ N. J. Hadley, ${ }^{169}$ S. Jabeen, ${ }^{169}$ G. Y. Jeng, ${ }^{169}$ R. G. Kellogg, ${ }^{169}$ J. Kunkle, ${ }^{169}$ A. C. Mignerey, ${ }^{169}$ S. Nabili, ${ }^{169}$ F. Ricci-Tam, ${ }^{169}$ M. Seidel, ${ }^{169}$ Y. H. Shin, ${ }^{169}$ A. Skuja ${ }^{169}$ S. C. Tonwar, ${ }^{169}$ K. Wong, ${ }^{169}$ D. Abercrombie, ${ }^{170}$ B. Allen, ${ }^{170}$ A. Baty,${ }^{170}$ R. Bi, ${ }^{170}$ S. Brandt,${ }^{170}$ W. Busza, ${ }^{170}$ I. A. Cali, ${ }^{170}$ M. D'Alfonso, ${ }^{170}$ G. Gomez Ceballos, ${ }^{170}$ M. Goncharov ${ }^{170}$ P. Harris, ${ }^{170}$ D. Hsu, ${ }^{170}$ M. Hu, ${ }^{170}$ M. Klute, ${ }^{170}$ D. Kovalskyi, ${ }^{170}$ Y.-J. Lee, ${ }^{170}$ P. D. Luckey, ${ }^{170}$ B. Maier, ${ }^{170}$ A. C. Marini, ${ }^{170}$ C. Mcginn, ${ }^{170}$ C. Mironov ${ }^{170}$ S. Narayanan, ${ }^{170}$ X. Niu, ${ }^{170}$ C. Paus, ${ }^{170}$ D. Rankin, ${ }^{170}$ C. Roland, ${ }^{170}$ G. Roland, ${ }^{170}$ Z. Shi, ${ }^{170}$ G. S. F. Stephans, ${ }^{170}$ K. Sumorok, ${ }^{170}$ K. Tatar, ${ }^{170}$ D. Velicanu, ${ }^{170}$ J. Wang, ${ }^{170}$ T. W. Wang, ${ }^{170}$ B. Wyslouch ${ }^{170}$ R. M. Chatterjee, ${ }^{171}$ A. Evans, ${ }^{171}$ S. Guts, ${ }^{171, a}$ P. Hansen, ${ }^{171}$ J. Hiltbrand, ${ }^{171}$ Sh. Jain, ${ }^{171}$ Y. Kubota, ${ }^{171}$ Z. Lesko, ${ }^{171}$ J. Mans,${ }^{171}$ M. Revering, ${ }^{171}$ R. Rusack,${ }^{171}$ R. Saradhy, ${ }^{171}$

N. Schroeder, ${ }^{171}$ M. A. Wadud, ${ }^{171}$ J. G. Acosta, ${ }^{172}$ S. Oliveros,,${ }^{172}$ K. Bloom, ${ }^{173}$ S. Chauhan, ${ }^{173}$ D. R. Claes, ${ }^{173}$ C. Fangmeier, ${ }^{173}$ L. Finco, ${ }^{173}$ F. Golf, ${ }^{173}$ R. Kamalieddin, ${ }^{173}$ I. Kravchenko, ${ }^{173}$ J. E. Siado, ${ }^{173}$ G. R. Snow, ${ }^{173, a}$ B. Stieger, ${ }^{173}$ W. Tabb, ${ }^{173}$ G. Agarwal, ${ }^{174}$ C. Harrington, ${ }^{174}$ I. Iashvili, ${ }^{174}$ A. Kharchilava, ${ }^{174}$ C. McLean, ${ }^{174}$ D. Nguyen, ${ }^{174}$ A. Parker, ${ }^{174}$ J. Pekkanen, ${ }^{174}$ S. Rappoccio, ${ }^{174}$ B. Roozbahani, ${ }^{174}$ G. Alverson, ${ }^{175}$ E. Barberis, ${ }^{175}$ C. Freer, ${ }^{175}$ Y. Haddad, ${ }^{175}$ A. Hortiangtham, ${ }^{175}$ G. Madigan, ${ }^{175}$ B. Marzocchi,${ }^{175}$ D. M. Morse, ${ }^{175}$ T. Orimoto, ${ }^{175}$ L. Skinnari, ${ }^{175}$

A. Tishelman-Charny, ${ }^{175}$ T. Wamorkar, ${ }^{175}$ B. Wang, ${ }^{175}$ A. Wisecarver, ${ }^{175}$ D. Wood, ${ }^{175}$ S. Bhattacharya, ${ }^{176}$ J. Bueghly, ${ }^{176}$ T. Gunter, ${ }^{176}$ K. A. Hahn, ${ }^{176}$ N. Odell, ${ }^{176}$ M. H. Schmitt, ${ }^{176}$ K. Sung, ${ }^{176}$ M. Trovato, ${ }^{176}$ M. Velasco, ${ }^{176}$ R. Bucci, ${ }^{177}$ N. Dev, ${ }^{177}$ R. Goldouzian, ${ }^{177}$ M. Hildreth, ${ }^{177}$ K. Hurtado Anampa ${ }^{177}$ C. Jessop,${ }^{177}$ D. J. Karmgard, ${ }^{177}$ K. Lannon,${ }^{177}$ W. Li, ${ }^{177}$ N. Loukas, ${ }^{177}$ N. Marinelli, ${ }^{177}$ I. Mcalister ${ }^{177}$ F. Meng, ${ }^{177}$ C. Mueller ${ }^{177}$ Y. Musienko, ${ }^{177, m m}$ M. Planer, ${ }^{177}$ R. Ruchti, ${ }^{177}$ P. Siddireddy, ${ }^{177}$ G. Smith, ${ }^{177}$ S. Taroni, ${ }^{177}$ M. Wayne, ${ }^{177}$ A. Wightman, ${ }^{177}$ M. Wolf, ${ }^{177}$ A. Woodard, ${ }^{177}$ J. Alimena, ${ }^{178}$ B. Bylsma, ${ }^{178}$ L. S. Durkin, ${ }^{178}$ B. Francis, ${ }^{178}$ C. Hill, ${ }^{178}$ W. Ji, ${ }^{178}$ A. Lefeld, ${ }^{178}$ T. Y. Ling, ${ }^{178}$ B. L. Winer,${ }^{178}$ G. Dezoort, ${ }^{179}$ P. Elmer, ${ }^{179}$ J. Hardenbrook, ${ }^{179}$ N. Haubrich, ${ }^{179}$ S. Higginbotham, ${ }^{179}$ A. Kalogeropoulos, ${ }^{179}$ S. Kwan, ${ }^{179}$ D. Lange, ${ }^{179}$ M. T. Lucchini, ${ }^{179}$ J. Luo, ${ }^{179}$ D. Marlow, ${ }^{179}$ K. Mei,${ }^{179}$ I. Ojalvo, ${ }^{179}$ J. Olsen, ${ }^{179}$ C. Palmer, ${ }^{179}$ P. Piroué, ${ }^{179}$

J. Salfeld-Nebgen, ${ }^{179}$ D. Stickland, ${ }^{179}$ C. Tully, ${ }^{179}$ Z. Wang, ${ }^{179}$ S. Malik, ${ }^{180}$ S. Norberg, ${ }^{180}$ A. Barker, ${ }^{181}$ V. E. Barnes, ${ }^{181}$

S. Das, ${ }^{181}$ L. Gutay, ${ }^{181}$ M. Jones, ${ }^{181}$ A. W. Jung, ${ }^{181}$ A. Khatiwada, ${ }^{181}$ B. Mahakud ${ }^{181}$ D. H. Miller, ${ }^{181}$ G. Negro, ${ }^{181}$ N. Neumeister, ${ }^{181}$ C. C. Peng, ${ }^{181}$ S. Piperov, ${ }^{181}$ H. Qiu,${ }^{181}$ J. F. Schulte, ${ }^{181}$ N. Trevisani,${ }^{181}$ F. Wang, ${ }^{181}$ R. Xiao, ${ }^{181}$ W. Xie, ${ }^{181}$ T. Cheng, ${ }^{182}$ J. Dolen, ${ }^{182}$ N. Parashar, ${ }^{182}$ U. Behrens, ${ }^{183}$ K. M. Ecklund, ${ }^{183}$ S. Freed, ${ }^{183}$ F. J. M. Geurts, ${ }^{183}$ M. Kilpatrick, ${ }^{183}$ 
Arun Kumar, ${ }^{183}$ W. Li, ${ }^{183}$ B. P. Padley, ${ }^{183}$ R. Redjimi, ${ }^{183}$ J. Roberts, ${ }^{183}$ J. Rorie, ${ }^{183}$ W. Shi, ${ }^{183}$ A. G. Stahl Leiton, ${ }^{183}$ Z. Tu, ${ }^{183}$ A. Zhang, ${ }^{183}$ A. Bodek, ${ }^{184}$ P. de Barbaro, ${ }^{184}$ R. Demina, ${ }^{184}$ J. L. Dulemba,${ }^{184}$ C. Fallon, ${ }^{184}$ T. Ferbel,${ }^{184}$ M. Galanti, ${ }^{184}$ A. Garcia-Bellido, ${ }^{184}$ O. Hindrichs, ${ }^{184}$ A. Khukhunaishvili, ${ }^{184}$ E. Ranken, ${ }^{184}$ R. Taus, ${ }^{184}$ B. Chiarito, ${ }^{185}$ J. P. Chou, ${ }^{185}$

A. Gandrakota, ${ }^{185}$ Y. Gershtein, ${ }^{185}$ E. Halkiadakis, ${ }^{185}$ A. Hart, ${ }^{185}$ M. Heindl, ${ }^{185}$ E. Hughes, ${ }^{185}$ S. Kaplan, ${ }^{185}$ I. Laflotte, ${ }^{185}$ A. Lath, ${ }^{185}$ R. Montalvo, ${ }^{185}$ K. Nash, ${ }^{185}$ M. Osherson, ${ }^{185}$ H. Saka, ${ }^{185}$ S. Salur, ${ }^{185}$ S. Schnetzer ${ }^{185}$ S. Somalwar, ${ }^{185}$ R. Stone ${ }^{185}$ S. Thomas, ${ }^{185}$ H. Acharya, ${ }^{186}$ A. G. Delannoy ${ }^{186}$ S. Spanier,${ }^{186}$ O. Bouhali, ${ }^{187, z z z}$ M. Dalchenko, ${ }^{187}$ M. De Mattia, ${ }^{187}$ A. Delgado, ${ }^{187}$ S. Dildick, ${ }^{187}$ R. Eusebi ${ }^{187}$ J. Gilmore, ${ }^{187}$ T. Huang, ${ }^{187}$ T. Kamon, ${ }^{187 \text {,aaaa }}$ S. Luo, ${ }^{187}$ S. Malhotra, ${ }^{187}$ D. Marley, ${ }^{187}$ R. Mueller, ${ }^{187}$ D. Overton, ${ }^{187}$ L. Perniè, ${ }^{187}$ D. Rathjens, ${ }^{187}$ A. Safonov, ${ }^{187}$ N. Akchurin, ${ }^{188}$ J. Damgov, ${ }^{188}$ F. De Guio, ${ }^{188}$ S. Kunori, ${ }^{188}$ K. Lamichhane, ${ }^{188}$ S. W. Lee, ${ }^{188}$ T. Mengke, ${ }^{188}$ S. Muthumuni,${ }^{188}$ T. Peltola, ${ }^{188}$ S. Undleeb, ${ }^{188}$ I. Volobouev, ${ }^{188}$ Z. Wang, ${ }^{188}$ A. Whitbeck, ${ }^{188}$ S. Greene, ${ }^{189}$ A. Gurrola, ${ }^{189}$ R. Janjam, ${ }^{189}$ W. Johns, ${ }^{189}$ C. Maguire, ${ }^{189}$ A. Melo, ${ }^{189}$ H. Ni, ${ }^{189}$ K. Padeken, ${ }^{189}$ F. Romeo, ${ }^{189}$ P. Sheldon, ${ }^{189}$ S. Tuo, ${ }^{189}$ J. Velkovska, ${ }^{189}$ M. Verweij, ${ }^{189}$ M. W. Arenton, ${ }^{190}$ P. Barria,${ }^{190}$ B. Cox,${ }^{190}$ G. Cummings, ${ }^{190}$ J. Hakala, ${ }^{190}$ R. Hirosky, ${ }^{190}$ M. Joyce, ${ }^{190}$ A. Ledovskoy, ${ }^{190}$ C. Neu, ${ }^{190}$ B. Tannenwald, ${ }^{190}$ Y. Wang, ${ }^{190}$ E. Wolfe,${ }^{190}$ F. Xia, ${ }^{190}$ R. Harr, ${ }^{191}$ P. E. Karchin, ${ }^{191}$ N. Poudyal,${ }^{191}$ J. Sturdy, ${ }^{191}$ P. Thapa ${ }^{191}$ T. Bose, ${ }^{192}$ J. Buchanan, ${ }^{192}$ C. Caillol,${ }^{192}$ D. Carlsmith, ${ }^{192}$ S. Dasu,${ }^{192}$ I. De Bruyn, ${ }^{192}$ L. Dodd,${ }^{192}$ F. Fiori, ${ }^{192}$ C. Galloni, ${ }^{192}$ B. Gomber, ${ }^{192, b b b b}$ H. He, ${ }^{192}$ M. Herndon, ${ }^{192}$ A. Hervé, ${ }^{192}$ U. Hussain, ${ }^{192}$ P. Klabbers, ${ }^{192}$ A. Lanaro, ${ }^{192}$ A. Loeliger, ${ }^{192}$ K. Long, ${ }^{192}$ R. Loveless,${ }^{192}$ J. Madhusudanan Sreekala, ${ }^{192}$ D. Pinna, ${ }^{192}$ T. Ruggles, ${ }^{192}$ A. Savin, ${ }^{192}$ V. Sharma, ${ }^{192}$ W. H. Smith, ${ }^{192}$ D. Teague, ${ }^{192}$ S. Trembath-reichert, ${ }^{192}$ and N. Woods ${ }^{192}$

(CMS Collaboration)

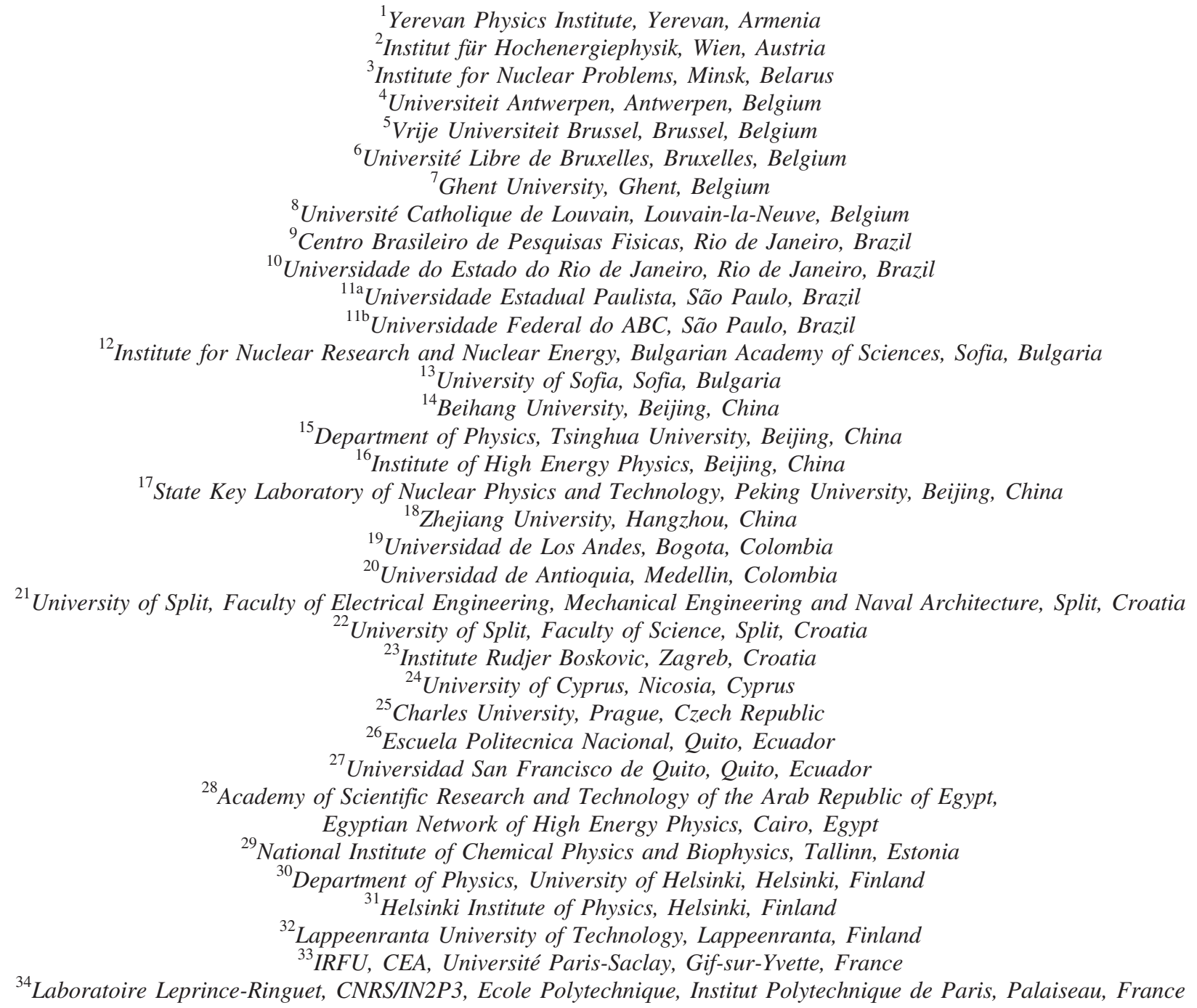


${ }^{35}$ Université de Strasbourg, CNRS, IPHC UMR 7178, Strasbourg, France

${ }^{36}$ Centre de Calcul de l'Institut National de Physique Nucleaire et de Physique des Particules, CNRS/IN2P3, Villeurbanne, France

${ }^{37}$ Université de Lyon, Université Claude Bernard Lyon 1, CNRS-IN2P3, Institut de Physique Nucléaire de Lyon, Villeurbanne, France

${ }^{38}$ Georgian Technical University, Tbilisi, Georgia

${ }^{39}$ Tbilisi State University, Tbilisi, Georgia

${ }^{40}$ RWTH Aachen University, I. Physikalisches Institut, Aachen, Germany

${ }^{41}$ RWTH Aachen University, III. Physikalisches Institut A, Aachen, Germany

${ }^{42}$ RWTH Aachen University, III. Physikalisches Institut B, Aachen, Germany

${ }^{43}$ Deutsches Elektronen-Synchrotron, Hamburg, Germany

${ }^{44}$ University of Hamburg, Hamburg, Germany

${ }^{45}$ Karlsruher Institut fuer Technologie, Karlsruhe, Germany

${ }^{46}$ Institute of Nuclear and Particle Physics (INPP), NCSR Demokritos, Aghia Paraskevi, Greece

${ }^{47}$ National and Kapodistrian University of Athens, Athens, Greece

${ }^{48}$ National Technical University of Athens, Athens, Greece

${ }^{49}$ University of Ioánnina, Ioánnina, Greece

${ }^{50}$ MTA-ELTE Lendület CMS Particle and Nuclear Physics Group, Eötvös Loránd University, Budapest, Hungary

${ }^{51}$ Wigner Research Centre for Physics, Budapest, Hungary

${ }^{52}$ Institute of Nuclear Research ATOMKI, Debrecen, Hungary

${ }^{53}$ Institute of Physics, University of Debrecen, Debrecen, Hungary

${ }^{54}$ Eszterhazy Karoly University, Karoly Robert Campus, Gyongyos, Hungary

${ }^{55}$ Indian Institute of Science (IISc), Bangalore, India

${ }^{56}$ National Institute of Science Education and Research, HBNI, Bhubaneswar, India

${ }^{57}$ Panjab University, Chandigarh, India

${ }^{58}$ University of Delhi, Delhi, India

${ }^{59}$ Saha Institute of Nuclear Physics, HBNI, Kolkata, India

${ }^{60}$ Indian Institute of Technology Madras, Madras, India

${ }^{61}$ Bhabha Atomic Research Centre, Mumbai, India

${ }^{62}$ Tata Institute of Fundamental Research-A, Mumbai, India

${ }^{63}$ Tata Institute of Fundamental Research-B, Mumbai, India

${ }^{64}$ Indian Institute of Science Education and Research (IISER), Pune, India

${ }^{65}$ Institute for Research in Fundamental Sciences (IPM), Tehran, Iran

${ }^{66}$ University College Dublin, Dublin, Ireland

${ }^{67 \mathrm{a} I N F N}$ Sezione di Bari, Bari, Italy

${ }^{67 \mathrm{~b}}$ Università di Bari, Bari, Italy

${ }^{67 \mathrm{c}}$ Politecnico di Bari, Bari, Italy

${ }^{68 \mathrm{a}}$ INFN Sezione di Bologna, Bologna, Italy

${ }^{68 \mathrm{~b}}$ Università di Bologna, Bologna, Italy

${ }^{69}$ INFN Sezione di Catania, Catania, Italy

${ }^{69 \mathrm{~b}}$ Università di Catania, Catania, Italy

${ }^{70 a}$ INFN Sezione di Firenze, Firenze, Italy

${ }^{70 \mathrm{~b}}$ Università di Firenze, Firenze, Italy

${ }^{71}$ INFN Laboratori Nazionali di Frascati, Frascati, Italy

${ }^{72 \mathrm{a}}$ INFN Sezione di Genova, Genova, Italy

${ }^{72 \mathrm{~b}}$ Università di Genova, Genova, Italy

${ }^{73 a}$ INFN Sezione di Milano-Bicocca, Milano, Italy

${ }^{73 b}$ Università di Milano-Bicocca, Milano, Italy

${ }^{74 \mathrm{a}}$ INFN Sezione di Napoli, Roma, Italy

${ }^{74 \mathrm{~b}}$ Università di Napoli 'Federico II', Roma, Italy

${ }^{74 c}$ Università della Basilicata, Roma, Italy

${ }^{74 \mathrm{~d}}$ Università G. Marconi, Roma, Italy

${ }^{75 a}$ INFN Sezione di Padova, Trento, Italy

${ }^{75 b}$ Università di Padova, Trento, Italy

${ }^{75 \mathrm{c}}$ Università di Trento, Trento, Italy

${ }^{76 a}$ INFN Sezione di Pavia, Pavia, Italy

${ }^{76 \mathrm{~b}}$ Università di Pavia, Pavia, Italy

${ }^{77 a}$ INFN Sezione di Perugia, Perugia, Italy

${ }^{77 \mathrm{~b}}$ Università di Perugia, Perugia, Italy

${ }^{78 a}$ INFN Sezione di Pisa, Pisa, Italy

${ }^{78 b}$ Università di Pisa, Pisa, Italy

${ }^{78 c}$ Scuola Normale Superiore di Pisa, Pisa, Italy 


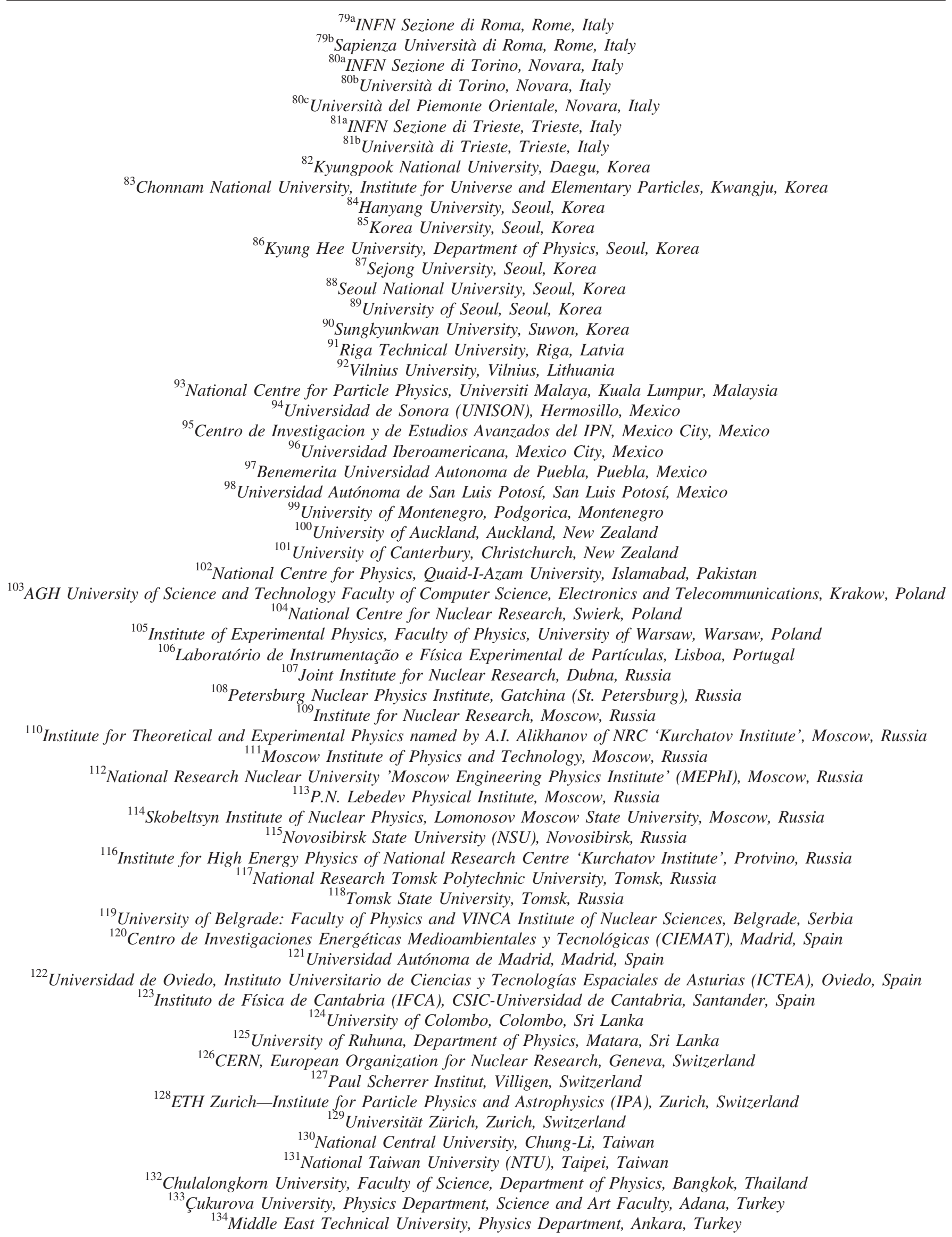




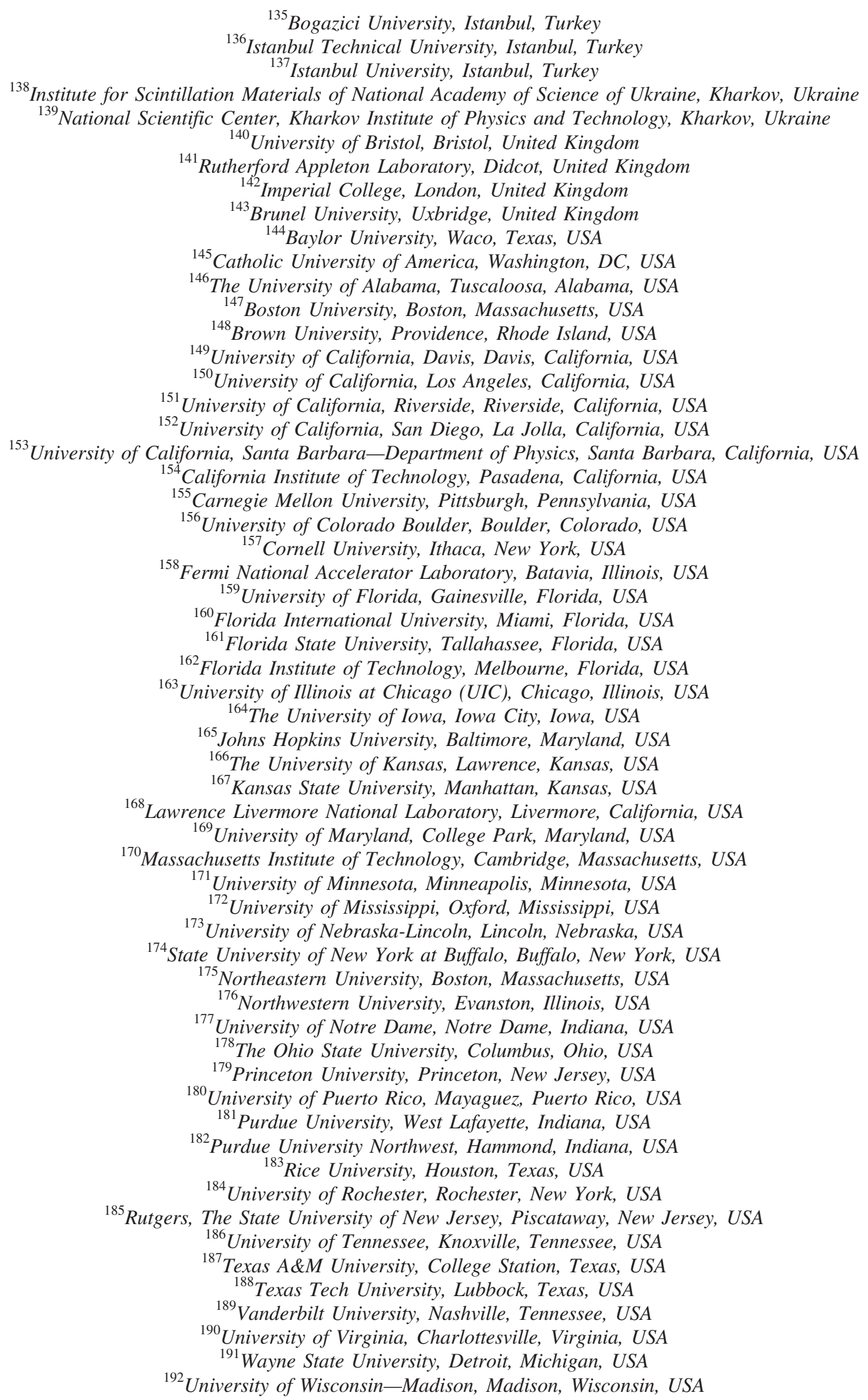


${ }^{\mathrm{a}}$ Deceased.

${ }^{\mathrm{b}}$ Also at Vienna University of Technology, Vienna, Austria.

${ }^{\mathrm{c}}$ Also at IRFU, CEA, Université Paris-Saclay, Gif-sur-Yvette, France.

${ }^{\mathrm{d}}$ Also at Universidade Estadual de Campinas, Campinas, Brazil.

${ }^{\mathrm{e}}$ Also at Federal University of Rio Grande do Sul, Porto Alegre, Brazil.

${ }^{\mathrm{f}}$ Also at UFMS.

${ }^{\mathrm{g}}$ Also at Universidade Federal de Pelotas, Pelotas, Brazil.

${ }^{\mathrm{h}}$ Also at Université Libre de Bruxelles, Bruxelles, Belgium.

${ }^{\mathrm{i}}$ Also at University of Chinese Academy of Sciences.

${ }^{\mathrm{j}}$ Also at Institute for Theoretical and Experimental Physics named by A.I. Alikhanov of NRC 'Kurchatov Institute', Moscow, Russia.

${ }^{\mathrm{k}}$ Also at Joint Institute for Nuclear Research, Dubna, Russia.

${ }^{1}$ Also at Helwan University, Cairo, Egypt.

${ }^{\mathrm{m}}$ Also at Zewail City of Science and Technology, Zewail, Egypt.

${ }^{\mathrm{n}}$ Also at Ain Shams University, Cairo, Egypt.

${ }^{\circ}$ Also at Purdue University, West Lafayette, Indiana, USA.

${ }^{\mathrm{p}}$ Also at Université de Haute Alsace, Mulhouse, France.

${ }^{\mathrm{q}}$ Also at Tbilisi State University, Tbilisi, Georgia.

${ }^{\mathrm{r}}$ Also at Erzincan Binali Yildirim University, Erzincan, Turkey.

${ }^{s}$ Also at CERN, European Organization for Nuclear Research, Geneva, Switzerland.

${ }^{t}$ Also at RWTH Aachen University, III. Physikalisches Institut A, Aachen, Germany.

"Also at University of Hamburg, Hamburg, Germany.

${ }^{v}$ Also at Brandenburg University of Technology, Cottbus, Germany.

${ }^{w}$ Also at Institute of Physics, University of Debrecen, Debrecen, Hungary.

${ }^{x}$ Also at Institute of Nuclear Research ATOMKI, Debrecen, Hungary.

${ }^{y}$ Also at MTA-ELTE Lendület CMS Particle and Nuclear Physics Group, Eötvös Loránd University, Budapest, Hungary.

${ }^{\mathrm{z}}$ Also at IIT Bhubaneswar, Bhubaneswar, India.

${ }^{\text {aa }}$ Also at Institute of Physics, Bhubaneswar, India.

${ }^{\mathrm{bb}}$ Also at Shoolini University, Solan, India.

${ }^{\mathrm{cc}}$ Also at University of Visva-Bharati, Santiniketan, India.

${ }^{\mathrm{dd}}$ Also at Isfahan University of Technology.

${ }^{e e}$ Also at INFN Sezione di Bari, Università di Bari, Politecnico di Bari, Bari, Italy.

${ }^{\mathrm{ff}}$ Also at Italian National Agency for New Technologies, Energy and Sustainable Economic Development.

${ }^{g g}$ Also at Centro Siciliano di Fisica Nucleare e di Struttura Della Materia.

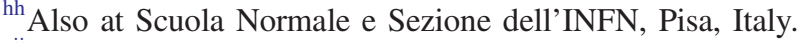

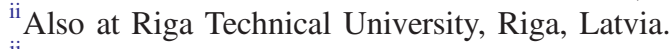

${ }^{j j}$ Also at Malaysian Nuclear Agency, MOSTI, Kajang, Malaysia.

${ }^{\mathrm{kk}}$ Also at Consejo Nacional de Ciencia y Tecnología, Mexico City, Mexico.

${ }^{11}$ Also at Warsaw University of Technology, Institute of Electronic Systems, Warsaw, Poland.

${ }^{\mathrm{mm}}$ Also at Institute for Nuclear Research, Moscow, Russia.

${ }^{\mathrm{nn}}$ Also at National Research Nuclear University 'Moscow Engineering Physics Institute' (MEPhI), Moscow, Russia.

${ }^{\text {oo }}$ Also at St. Petersburg State Polytechnical University, St. Petersburg, Russia.

${ }^{\mathrm{pp}}$ Also at University of Florida, Gainesville, Florida, USA.

${ }^{\mathrm{qq}}$ Also at Imperial College, London, United Kingdom.

${ }^{\mathrm{rr}}$ Also at P.N. Lebedev Physical Institute, Moscow, Russia.

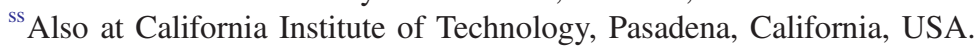

"Also at Budker Institute of Nuclear Physics, Novosibirsk, Russia.

${ }^{u u}$ Also at Faculty of Physics, University of Belgrade, Belgrade, Serbia.

${ }^{v v}$ Also at Università degli Studi di Siena, Siena, Italy.

${ }^{\text {ww }}$ Also at INFN Sezione di Pavia, Università di Pavia, Pavia, Italy.

${ }^{\mathrm{xx}}$ Also at National and Kapodistrian University of Athens, Athens, Greece.

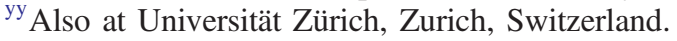

${ }^{z z}$ Also at Stefan Meyer Institute for Subatomic Physics, Vienna, Austria.

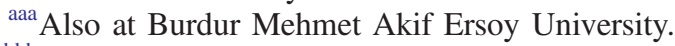

${ }^{\mathrm{bbb}}$ Also at Adiyaman University, Adiyaman, Turkey.

${ }^{c c c}$ Also at Şırnak University.

ddd Also at Department of Physics, Tsinghua University, Beijing, China.

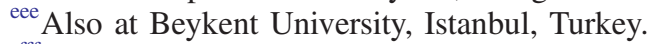

${ }^{\mathrm{fff}}$ Also at Istanbul Aydin University, Application and Research Center for Advanced Studies (App. \& Res. Cent. for Advanced Studies). 


\footnotetext{
${ }^{\text {ggg }}$ Also at Mersin University, Mersin, Turkey.

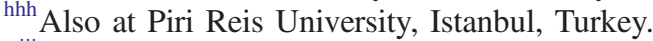

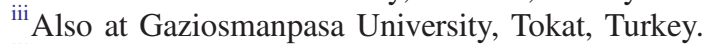

${ }^{\mathrm{jij}}$ Also at Ozyegin University, Istanbul, Turkey.

${ }^{k k k}$ Also at Izmir Institute of Technology, Izmir, Turkey.

${ }^{111}$ Also at Marmara University, Istanbul, Turkey.

${ }^{\mathrm{mmm}}$ Also at Kafkas University, Kars, Turkey.

${ }^{n n n}$ Also at Istanbul Bilgi University, Istanbul, Turkey.

${ }^{000}$ Also at Hacettepe University, Ankara, Turkey.

${ }^{p p p}$ Also at Vrije Universiteit Brussel, Brussel, Belgium.

${ }^{\mathrm{qqq}}$ Also at School of Physics and Astronomy, University of Southampton, Southampton, United Kingdom.

${ }^{\mathrm{rrr}}$ Also at IPPP Durham University.

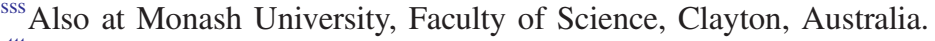

${ }^{t t t}$ Also at Bethel University, St. Paul, Minneapolis, USA.

${ }^{u u u}$ Also at Karamanoğlu Mehmetbey University, Karaman, Turkey.

${ }^{\mathrm{v} v \mathrm{v}}$ Also at Bingol University, Bingol, Turkey.

${ }^{\text {www }}$ Also at Georgian Technical University, Tbilisi, Georgia.

${ }^{\mathrm{xxx}}$ Also at Sinop University, Sinop, Turkey.

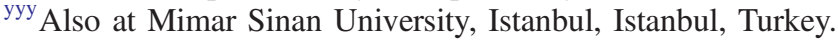

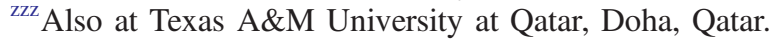

${ }^{\text {aaa }}$ Also at Kyungpook National University, Daegu, Korea.

${ }^{b b b b}$ Also at University of Hyderabad, Hyderabad, India.
} 